\title{
$p$-adic path set fractals and arithmetic
}

\author{
William C. Abram ${ }^{1}$ and Jeffrey C. Lagarias ${ }^{2}$
}

\begin{abstract}
This paper considers a class of closed subsets of the $p$-adic integers $\mathbb{Z}_{p}$ obtained by graph-directed constructions analogous to that of Mauldin and Williams over the real numbers. These sets are characterized as the collection of those $p$-adic integers whose points have $p$-adic expansions describable by paths in the graph of a finite automaton issuing from a distinguished initial vertex. This paper shows that this class of sets is closed under the arithmetic operations of addition and multiplication by $p$-integral rational numbers $r \in \mathbb{Q} \cap \mathbb{Z}_{p}$. In addition the Minkowski sum (under $p$-adic addition) of two sets in this class is shown to be another set in this class. These results represent purely $p$-adic phenomena in that analogous closure properties do not hold over the real numbers. We also show the existence of computable formulas for the Hausdorff dimensions of such sets.
\end{abstract}

Mathematics Subject Classification (2010). Primary 11K55; Secondary 11S82, 28A80, 37B10.

Keywords. $p$-adic arithmetic, finite automata, graph-directed systems, Hausdoff dimension.

\section{Contents}

1 Introduction . . . . . . . . . . . . . . . . . . . 46

2 Relation to geometric graph-directed constructions . . . . . . . . . . . 52

3 Hausdorff dimension of $p$-adic path set fractals . . . . . . . . . . . . 59

$4 p$-adic addition and path set fractals .............. 65

5 -adic multiplication and path set fractals . . . . . . . . . . . 70

6 Examples ........................ 75

7 Concluding remarks . . . . . . . . . . . . . . . . . . 79

References ..................... 80

\footnotetext{
${ }^{1}$ W. C. Abram acknowledges the support of an NSF Graduate Research Fellowship.

${ }^{2}$ J. C. Lagarias was partially supported by NSF grant DMS- 1101373.
} 


\section{Introduction}

This paper studies a distinguished collection $\ell\left(\mathbb{Z}_{p}\right)$ of closed subsets of the $p$-adic integers $\mathbb{Z}_{p}$, whose members $Y$ are specified as sets of $p$-adic integers whose $p$-adic expansions are given by infinite labeled paths starting from a fixed initial state of a finite automaton, with edge labels specifying $p$-adic digits. We term such sets $p$-adic path set fractals, because they generally have non-integer Hausdorff dimension, and because they may be constructed geometrically in a fashion analogous to that of the (real-valued) geometric graph-directed fractals of Mauldin and Williams [19] and [20], as explained in Section 2.

The set of edge-labeled infinite paths in the graph of an automaton which start from a fixed state can be studied abstractly in terms of one-sided symbolic dynamics, as we consider elsewhere ([2]). Each such set defines a subset $X_{\mathscr{E}}(v)$ of the symbol space $\mathcal{A}^{\mathbb{N}}$, where $\mathcal{A}$ is a finite symbol alphabet, which is specified by a presentation $(\mathcal{G}, v)$, in which $\mathcal{E}$ is a labeled directed graph with edges labeled by elements of $\mathcal{A}$, and $v$ is a marked initial vertex of $\mathscr{E}$. We call $X_{\mathscr{E}}(v)$ an (abstract) path set. Path sets are closed subsets of the compact set $\mathscr{A}^{\mathbb{N}}$ endowed with the product topology, but they are generally not invariant under the (one-sided) shift

$$
\sigma: \mathcal{A}^{\mathbb{N}} \longrightarrow \mathcal{A}^{\mathbb{N}}
$$

given by

$$
\sigma\left(\alpha_{0}, \alpha_{1}, \alpha_{2}, \ldots\right)=\left(\alpha_{1}, \alpha_{2}, \alpha_{3}, \ldots\right) .
$$

The collection of path sets is closed under set union and set intersection, but is not closed under complementation inside the symbol space $\mathcal{A}^{\mathbb{N}}$.

A p-adic path set fractal $Y$ is the image of an abstract path set embedded as a geometric object inside a $p$-adic space $\mathbb{Z}_{p}$, using the symbol sequence to obtain the $p$-adic digit labeling. More precisely,

$$
Y=f_{p}(X \mathscr{E}(v)) \subset \mathbb{Z}_{p},
$$

where

$$
f_{p}: \mathcal{A}^{\mathbb{N}} \longrightarrow \mathbb{Z}_{p}
$$

is a (continuous) map that sends a symbol sequence to a set of $p$-adic digits, using a digit assignment map

$$
\bar{f}_{p}: \mathcal{A} \rightarrow\{0,1, \ldots, p-1\} .
$$

The digit assignment map $\bar{f}_{p}$ need not be one-to-one, consequently a given abstract path set $X \mathscr{E}(v)$ has embeddings into $\mathbb{Z}_{p}$ for every prime $p$. Moreover it typically has different embeddings into a fixed $\mathbb{Z}_{p}$, giving rise to different $p$-adic path set fractals. In the reverse direction any given $p$-adic path set fractal can be obtained as the image of different abstract path sets using different digit assignment maps. However any $p$-adic path set fractal $Y$ can always be obtained by a one-to-one embedding from a suitably chosen path set on the fixed alphabet $\mathcal{A}=\{0,1, \ldots, p-1\}$, see 
Proposition 2.9 below. We call the data $\left(\bar{f}_{p}, \mathcal{E}, v\right)$ a presentation of the $p$-adic path set fractal, and write

$$
Y \stackrel{\text { def }}{=}\left(\bar{f}_{p}, \mathcal{E}, v\right) \text {. }
$$

The initial part of this paper gives a formula for the Hausdorff dimension of such a set $Y$ in terms of the spectral radius of the adjacency matrix of an underlying automaton of a suitable presentation of $Y$, described in Section 3. The Hausdorff dimension of $Y=f_{p}\left(X_{\mathscr{E}}(v)\right)$ depends on the underlying path set $X_{\mathscr{E}}(v)$, the value of $p$, and on the digit assignment map $f_{p}$. We obtain the formula by relating the $p$-adic constructions of this paper to the real number constructions of Mauldin and Williams [20], which permit carrying over their formulas for Hausdorff dimension of (real) graph-directed fractals to the $p$-adic case.

The main object of this paper is to show that the collection of $p$-adic path set fractals $\mathscr{C}\left(\mathbb{Z}_{p}\right)$ is closed under the following operations using $p$-adic arithmetic:

(1) $p$-adic addition of a rational number $r \in \mathbb{Q} \cap \mathbb{Z}_{p}$ (such $r$ are called $p$-integral);

(2) $p$-adic multiplication by a $p$-integral rational number $r$;

(3) set-valued addition (Minkowski sum) of two $p$-adic path sets, using $p$-adic addition.

These closure results represent purely $p$-adic phenomena in the sense that analogous closure results for applying real arithmetic operations ${ }^{1}$ fail to hold for MauldinWilliams graph-directed fractals over the real numbers. We show that the finite automata describing the new sets given by these operations are effectively computable; these new automata depend on the input automata and on the value of $p$ in a complicated way having a number-theoretic flavor.

1.1. Results. As a preliminary to $p$-adic results, in Section 2 we review the MauldinWilliams construction of graph-directed fractals over the real numbers. We then formulate an alternate definition of $p$-adic path set fractals, defining them geometrically as sets given by a solution of a set-valued functional equation using $p$-adic contracting maps (Theorem 2.6 and Definition 2.7). That is, they are characterized as a set-valued fixed point of a $p$-adic graph-directed fractal construction. Then we establish the equivalence of this geometric definition to the symbolic dynamics definition given above, in terms of $p$-adic expansions describable by a finite automaton, as the image in $\mathbb{Z}_{p}$ of a path set under a digit assignment map (Theorem 2.10).

To state results, we need some additional terminology on presentations.

(1) A presentation $Y \stackrel{\text { def }}{=}\left(\bar{f}_{p}, \mathcal{E}, v\right)$ of a $p$-adic path set fractal is injective if the digit assignment map $\bar{f}_{p}$ is one-to-one.

(2) A path set presentation $(\mathcal{E}, v)$ is right-resolving if the directed graph underlying $\mathcal{Y}$ has the property that at each vertex of the graph, all exiting edges have different labels.

\footnotetext{
${ }^{1}$ That is, for addition to and multiplication of a set by rational numbers, or for the Minkowski sum of two sets.
} 
(3) A path set presentation $(\mathcal{G}, v)$ is reachable if every vertex in $\mathcal{G}$ can be reached by a directed path from $v$.

All $p$-adic path set fractals $Y$ have presentations that are injective, right-resolving and reachable, see Proposition 2.9. We call any such presentation a standard presentation. In a standard presentation one may always choose to relabel the symbol alphabet $\mathcal{A}=\{0,1,2, \ldots, p-1\}$, and choose the digit assignment map to be the identity map.

Our first result concerns the Hausdorff dimension $d_{H}(Y)$ of a $p$-adic path set fractal $Y=f_{p}\left(X_{\mathscr{E}}(v)\right)$. We show that $d_{H}(Y)$ is directly computable from a suitable presentation of $Y$ as a $p$-adic path set fractal, and is of an expected form.

Theorem 1.1 (Hausdorff dimension). Let $Y$ belong to $\left.\mathcal{C}_{p}\right)$ and let $Y \stackrel{\text { def }}{=}\left(\bar{f}_{p}, \mathcal{E}, v_{0}\right)$ be any standard presentation. Then its Hausdorff dimension $d_{H}(Y)$ is given by

$$
d_{H}(Y)=\log _{p} \sigma(A(\mathscr{E}))=\frac{\log \sigma(A(\mathscr{E}))}{\log p}
$$

where $\sigma(A)$ denotes the spectral radius of the adjacency matrix $A=A(\mathscr{E}) \stackrel{\text { def }}{=}\left[a_{i, j}\right]$ of $\mathcal{G}$, in which $a_{i, j}$ counts the number of directed edges from vertex $i$ to vertex $j$ of the underlying directed graph of $\mathcal{E}$.

This result is proved in Section 3, where it is deduced from a result relating these sets to real number graph-directed fractals (Theorem 3.1), where a similar Hausdorff dimension formula has long been known. We use the fact that Hausdorff dimension is preserved under the map taking a $p$-adic expansion of a $p$-adic integer to the base $p$ radix expansion of a real number. The corresponding real number objects are Mauldin-Williams connstruction sub-objects. General properties of Hausdorff dimension can be found in Rogers [23] or Falconer [10].

The main results of this paper concern $p$-adic arithmetic operations applied to $p$-adic path set fractals. We begin with addition of $p$-adic rationals.

Theorem 1.2 (Closure under rational addition). Let $Y$ belong to $\left.\ell_{(} \mathbb{Z}_{p}\right)$. Then for any $p$-integral rational number $r \in \mathbb{Q} \cap \mathbb{Z}_{p}$, the additively shifted set

$$
Y+r \stackrel{\text { def }}{=}\{y+r: y \in Y\}
$$

has $Y+r \in \mathcal{C}\left(\mathbb{Z}_{p}\right)$.

The proof of this result is constructive and shows that given a standard presentation $Y \stackrel{\text { def }}{=}\left(\bar{l}_{p}, \mathcal{E}, v\right)$ one can directly compute from it a standard presentation $Y+r \stackrel{\text { def }}{=}$ $\left(\bar{l}_{p}, \mathscr{\ell}^{\prime}, v^{\prime}\right)$. The new presentation depends on both $r \in \mathbb{Q}$ and the value of $p$.

Theorem 1.2 also follows as a special case of the following result. 
Theorem 1.3 (Closure under Minkowski sum). Let $Y_{1}, Y_{2} \in \mathcal{C}\left(\mathbb{Z}_{p}\right)$ be two p-adic path set fractals in $\mathbb{Z}_{p}$. Then their Minkowski sum-set

$$
Y_{1}+Y_{2} \stackrel{\text { def }}{=}\left\{y_{1}+y_{2}: y_{1} \in Y_{1}, y_{2} \in Y_{2}\right\}
$$

has $Y_{1}+Y_{2} \in \mathcal{C}\left(\mathbb{Z}_{p}\right)$.

The proof of this result is constructive and shows that given standard presentations for $Y_{1} \stackrel{\text { def }}{=}\left(\bar{l}_{p}, \boldsymbol{E}_{1}, v_{1}\right)$ and $Y_{2} \stackrel{\text { def }}{=}\left(\bar{l}_{p}, \boldsymbol{E}_{2}, v_{2}\right)$ one can directly construct a (not necessarily standard) presentation $Y_{1}+Y_{2}=\left(\bar{l}_{p}, \mathscr{g}_{3}, v_{3}\right)$. In the given construction the underlying path set presentation $\left(\mathscr{E}_{3}, v_{3}\right)$ produced is not necessarily right-resolving. However there exist standard algorithms to convert any given path set presentation to one that is right-resolving, see [2], Theorem 3.2.

The statement of Theorem 1.2 can be recovered from Theorem 1.3 as the special case that the set $Y_{2}$ is a one-element set, using the easy observation that the only path sets in $\mathbb{Z}_{p}$ consisting of a single element are those where this element is a rational $r \in \mathbb{Q} \cap \mathbb{Z}_{p}$ (Theorem 2.11). However the presentation obtained by the construction of Theorem 1.3 is not necessarily right-resolving, while the construction given in the proof of Theorem 1.2 is right-resolving.

We next consider multiplication by $p$-adic rationals.

Theorem 1.4 (Closure under rational multiplication). Let $Y$ belong to $\mathscr{C}\left(\mathbb{Z}_{p}\right)$. Then for any rational number $r \in \mathbb{Q} \cap \mathbb{Z}_{p}$, the dilated set

$$
r Y \stackrel{\text { def }}{=}\{r y: y \in Y\}
$$

has $r Y \in \mathcal{C}\left(\mathbb{Z}_{p}\right)$.

We prove this result in Section 5. This proof is constructive in the same sense as Theorem 1.1; given a standard presentation for $Y$ there is (in principle) an algorithm to find a standard presentation for $r Y$. Theorem 1.4 is obtained by concatenation of constructions for several special cases, as follows.

(1) $r=M$ is a positive integer with $\operatorname{gcd}(p, M)=1$. A positive integer has an infinite $p$-adic expansion with a finite pre-period and a periodic part with all digits 0 .

(2) $r=\frac{1}{M}$ is the inverse of a positive integer $M$ with $\operatorname{gcd}(p, M)=1$.

(3) $r=-1$. Note that -1 has a purely periodic nonterminating $p$-adic expansion of period 1:

$$
-1=\sum_{k=0}^{\infty}(p-1) p^{k}=(\ldots, p-1, p-1, p-1)_{p} .
$$

(4) $r=p^{k}$, for $k \geq 1$. 
Finally we note that, at the level of symbolic dynamics, the arithmetic operations are not compatible with the one-sided shift operation. That is, if $Y$ is a $p$-adic path set which is invariant under the one-sided shift $\sigma_{p}: \mathbb{Z}_{p} \rightarrow \mathbb{Z}_{p}$ defined by

$$
\sigma_{p}\left(\sum_{j=0}^{\infty} \alpha_{j} p^{j}\right) \stackrel{\text { def }}{=} \sum_{j=0}^{\infty} \alpha_{j+1} p^{j},
$$

then in general the sets $Y+r, r Y$ (for $p$-integral rational $r$ ) will not be invariant under the one-sided shift $\sigma_{p}$.

1.2. Extensions and generalizations. In Theorem 1.1 we give a formula for the Hausdorff dimension of a $p$-adic path set fractal $Y$ in terms of the spectral radius of a nonnegative integer matrix specifying the graph of the underlying path set. This formula might initially appear unnecessary in the context of the $p$-adic arithmetic operations studied, because given any set $X \subset \mathbb{Z}_{p}$ and any nonzero $\alpha \in \mathbb{Z}_{p}$, rational or not, the sets $X, X+\alpha$ and $\alpha X$ all have the same Hausdorff dimension. This fact follows since both sets $X+\alpha$ and $\alpha X$ are images of $X$ under bi-Lipschitz mappings in the $p$-adic metric. In this context, the usefulness of Theorem 1.1 lies rather in the opposite direction: using it, the known equality of Hausdorff dimensions yields the equality of the spectral radii of two quite different appearing nonnegative matrices, usually of different sizes.

It is of interest that the constructions of this paper will, when given a fixed set $X$ as input, by varying $r$ yield infinite classes of nonnegative integer matrices having a fixed spectral radius. This spectral radius always equals the largest real eigenvalue, which is a particular type of real algebraic integer called a weak Perron number. Here a weak Perron number is a (positive real) $n$-th root of a Perron number for some $n \geq 1$, cf. Lind [15] and [16]. A Perron number is a real algebraic integer $\beta>1$, all of whose algebraic conjugates $\beta^{\sigma}$ are smaller in modulus, i.e. $\left|\beta^{\sigma}\right|<\beta$. Such classes of matrices may be worth further study in connection with number theoretic problems, see Section 7.

The constructions of this paper can be combined with other operations which preserve the property of being a $p$-adic path set fractal but which do change the Hausdorff dimension. For example, path sets are closed under set union and set intersection ([2], Theorem 1.2), with the new path set presentations being effectively computable from the given ones. Set union changes the Hausdorff dimension in a predictable way, with the new set having dimension equal to the maximum of the two dimensions, however set intersection changes Hausdorff dimension in seemingly unpredictable ways. Given presentations of $p$-adic path set fractals $Y_{1}$ and $Y_{2}$ one can, in principle, compute the Hausdorff dimension of intersections of additive and multiplicative translates of these sets, such as $Y_{1} \bigcap\left(Y_{2}+r\right)$ and $Y_{1} \bigcap\left(r Y_{2}\right)$. This study was undertaken to answer questions of this kind that arose in connection with a problem of Erdős, see Erdős [9], and papers [13] and [3] of the authors. Computed examples in [3] illustrate that the Hausdorff dimensions of sets $Y \cap(Y+r)$ and 
$Y \cap r Y$ vary with $r$, and the dependence on $r$ of these Hausdorff dimensions appears to be extremely complicated, with interesting structure.

The class $\ell\left(\mathbb{Z}_{p}\right)$ of $p$-adic path set fractals are closed under another operation: decimation, i.e. extracting a fixed arithmetic progression of their $p$-adic digits. We set

$$
\psi_{j, m}\left(\alpha_{0}, \alpha_{1}, \alpha_{2}, \ldots\right)=\left(\alpha_{j}, \alpha_{j+m}, \alpha_{j+2 m}, \alpha_{j+3 m}, \ldots\right),
$$

and the define the $(j, m)$-decimated set

$$
\psi_{j, m}(Y)=\left\{\psi_{j, m}(x): x \in Y\right\} .
$$

The fact that $\psi_{j, m}(Y)$ set belongs to $\left.C_{\mathbb{Z}_{p}}\right)$ if $Y$ does follows at the path set level from [2], Theorem 1.5, which shows that a presentation of $\psi_{j, m}(Y)$ is effectively computable given a standard presentation of $Y$. Study of the effect of decimation operations on Hausdorff dimension of the images seems an interesting topic for further research.

There are a number of further directions in which the results may be generalized. The methods of this paper apply to arithmetic operations applied to the $g$-adic numbers for arbitrary $g \geq 2$, as defined by Mahler [18]. As a topological space one has $\mathbb{Z}_{g}=\prod_{p \mid g} \mathbb{Z}_{p}$. However when $g$ contains prime powers one would use a $g$-adic expansion corresponding to the alphabet $\mathcal{A}=\{0,1, \ldots, g-1\}$.

A second generalization is to allow sets in the $p$-adic numbers $\mathbb{Q}_{p}$, in which case addition or multiplication of arbitrary rational numbers would be permitted. One may also generalize the notion of $p$-adic path sets to higher dimensions, which would correspond to $\left(\mathbb{Z}_{p}\right)^{d}$. In this case one may investigate various relaxations of the overlap conditions imposed in the Mauldin-Williams construction. In the real number analogue $\mathbb{R}^{n}$ results have been obtained by Ngai and Wang [22], Das and Ngai [6], and Ngai, Wang, and Dong [21].

1.3. Contents of the paper. Section 2 recalls Mauldin-Williams constructions, gives two equivalent characterizations of $p$-adic path set fractals, and determines all $Y \in \mathcal{C}\left(\mathbb{Z}_{p}\right)$ that contain exactly one element. Section 3 gives formulas for Hausdoff dimension of path set fractals. Section 4 proves results on addition of rational numbers to $p$-adic path set fractals, and on set-valued addition of two $p$-adic path set fractals. Section 5 proves results on multiplication of $p$-adic path set fractals by rational numbers. Section 6 presents examples illustrating the results. Section 7 makes concluding remarks about how the constructions of this paper relate to integer matrices.

Acknowledgments. The authors thank the reviewer for helpful comments. 


\section{Relation to geometric graph-directed constructions}

We first describe the Mauldin-Williams geometric graph-directed construction in the real number case. Then we formulate a (restricted) $p$-adic analogue to it, and show that all $p$-adic path set fractals are obtained by such a construction, and conversely. The final subsection characterizes those $p$-adic path set fractals containing exactly one element.

2.1. Mauldin-Williams graph-directed constructions. In the 1980s Mauldin and Williams [20] introduced general graph-directed constructions of fractal sets over the real numbers, and computed their Hausdorff dimensions, see also Edgar [7], Chapter 4, and Edgar and Golds [8]. We follow the notation established in Mauldin and Williams [20].

Definition 2.1. A geometric graph-directed construction in $\mathbb{R}^{m}$ consists of the following data.

(G1) A finite sequence of nonoverlapping, ${ }^{2}$ compact subsets $J_{1}, \ldots, J_{n}$ of $\mathbb{R}^{m}$, such that each $J_{i}$ has a nonempty interior.

(G2) A directed graph $G$ with vertex set consisting of the integers $1, \ldots, n$, such that for each pair $(i, j)$ there is at most one directed edge from $i$ to $j$. Additionally, this graph must have the following properties:

(a) for each vertex $i$, there must be at least one exit edge, i.e. some $j$ such that $(i, j) \in G$;

(b) the underlying undirected graph must be connected.

(G3) For each graph edge $(i, j)$ there is assigned a similarity map

$$
T_{i, j}: \mathbb{R}^{m} \longrightarrow \mathbb{R}^{m},
$$

with similarity ratio $t_{i, j}$ such that:

(a) for each $i,\left\{T_{i, j}\left(J_{j}\right) \mid(i, j) \in G\right\}$ is a nonoverlapping family and

$$
J_{i} \supseteq \bigcup\left\{T_{i, j}\left(J_{j}\right) \mid(i, j) \in G\right\} ;
$$

(b) if the path component of $G$ rooted at the vertex $i_{1}$ is a cycle:

$$
\left[i_{1}, \ldots, i_{q}, i_{q+1}=i_{1}\right],
$$

then these satisfy the contraction condition

$$
\prod_{k=1}^{q} t_{i_{k}, i_{k+1}}<1 .
$$

\footnotetext{
${ }^{2}$ Sets $J_{1}$ and $J_{2}$ overlap if their intersection has a nonempty interior.
} 
Note that in this construction the similarity maps $T_{i, j}$ will be applied to map sets in the reverse direction to that of the edges of $G$.

Now for each $i$ let $\mathcal{K}\left(J_{i}\right)$ denote the space of compact subsets of $J_{i}$, with the Hausdorff metric, $\rho_{H}$. Mauldin and Williams prove the following result.

Proposition 2.2. For each geometric graph-directed construction, there exists a unique vector of compact sets, $\left(K_{1}, \ldots, K_{n}\right) \in \prod_{i=1}^{n} \mathcal{K}\left(J_{j}\right)$ such that, for each $i$,

$$
K_{i}=\bigcup\left\{T_{i, j}\left(K_{j}\right) \mid(i, j) \in G\right\} .
$$

Proof. This is proved by Mauldin and Williams ([20], Theorem 1, p. 812), using the results of Hutchinson [12].

The construction object $K$ is then defined by

$$
K=\bigcup_{i=1}^{n} K_{i} .
$$

The individual $K_{j}$ are the construction sub-objects.

Our definition of $p$-adic path sets will correspond to all possible construction sub-objects $K_{v}$ in the Mauldin-Williams construction.

Associated to the graph $G$ is an $n \times n$ construction matrix $A=A(G)$ (with $n=|V(G)|)$ given by

$$
A=A(G) \stackrel{\text { def }}{=}\left[t_{i, j}\right]_{1 \leq i, j \leq n},
$$

where $t_{i, j}$ is defined to be zero if $(i, j) \notin G$. Now for $\beta>0$, set

$$
A_{\beta}=\left[\left(t_{i, j}\right)^{\beta}\right]_{1 \leq i, j \leq n},
$$

and let

$$
\Phi(\beta) \stackrel{\text { def }}{=} \text { Spectral radius of } A_{\beta} .
$$

This is the largest non-negative eigenvalue of $A_{\beta}$, by the Perron-Frobenius theorem. Mauldin and Williams [20], Theorem 2, observe that for each construction matrix, one has

(1) $\Phi(0) \geq 1$,

(2) $\Phi(\beta)$ is a continuous, strictly decreasing function of $\beta \geq 0$,

(3) $\lim _{\beta \rightarrow \infty} \Phi(\beta)=0$.

It follows that there is a unique value $\alpha \geq 0$ such that $\Phi(\alpha)=1$. They term this value the matrix dimension of the matrix $A=A_{G}$.

Mauldin and Williams determine the Hausdorff dimension of the construction object $K$ and also of its individual sub-objects $K_{j}$. For the construction object $K$ it is given as follows. 
Proposition 2.3. For each geometric graph-directed construction, the Hausdorff dimension of $K$, the construction object, is $\alpha$, where $\alpha$ is the matrix dimension of the construction matrix $A(G)=\left[t_{i, j}\right]_{1 \leq i, j \leq n}$, with $n=|V(G)|$. That is, it is the unique value $\alpha \geq 0$ such that the spectral radius $\sigma\left(A_{\alpha}\right)=1$, where $A_{\beta} \stackrel{\text { def }}{=}\left[\left(t_{i, j}\right)^{\beta}\right]_{1 \leq i, j \leq n}$ for $\beta>0$.

Proof. This is Theorem 3 of Mauldin and Williams [20].

Hausdorff dimension formulas for construction sub-objects $K_{v}$ involve the strongly connected components of the directed graph $G$, and use matrices which are square submatrices of $A(G)$, extracting specified rows and corresponding columns. A strongly connected component of a directed graph $G$ is a maximal subgraph that is strongly connected (i.e. each vertex in the component is reachable from each other vertex in it by a directed path.) We let $S C(G)$ denote the set of strongly connected components of the connected graph $G$. There is a natural partial ordering on $S C(G)$ which sets $H_{1} \preceq H_{2}$ provided there is a directed path in $G$ from a vertex in $H_{1}$ to one in $H_{2}$. We let $\alpha_{H}$ denote the matrix dimension of the square submatrix $A_{H}$ of the construction matrix $A_{G}$ corresponding to the strongly connected component $H$ of $G$.

Proposition 2.4. For each geometric graph-directed construction, the following hold.

(1) The Hausdorff dimension of $K$, the construction object, is $\alpha$, where

$$
\alpha=\max \left\{\alpha_{H} \mid H \in S C(G)\right\} .
$$

Furthermore the set $K$ has positive $\sigma$-finite $\mathscr{H}^{\alpha}$ measure.

(2) The Hausdorff dimension of each construction sub-object $K_{j}$ is $\alpha_{j}$, where

$$
\alpha_{j} \stackrel{\text { def }}{=} \max \left\{\alpha_{H} \mid H \in C_{j}\right\},
$$

where $C_{j}$ is the set of strongly connected components of $G$ reachable from vertex $j$. The sub-object $K_{j}$ has positive $\sigma$-finite $\alpha_{j}$-dimensional Hausdorffmeasure. This measure is finite if and only if $\left\{H \in C_{j} \mid \alpha_{H}=\alpha_{j}\right\}$ consists of (pairwise) incomparable elements in the partial order $\preceq$ on $S C(G)$.

Proof. This statement combines Theorems 4 and 5 of Mauldin and Williams [20], p. 814 and p. 824 .

2.2. $p$-adic graph-directed constructions. We formulate a $p$-adic variant of the Mauldin-Williams construction inside the compact set $\mathbb{Z}_{p}$ as follows. 
Definition 2.5. A (restricted) p-adic graph-directed construction on the $p$-adic integers $\mathbb{Z}_{p}$ consists of the following data.

(P1) A finite sequence of (identical) initial sets $J_{i}=\mathbb{Z}_{p}$, for $1 \leq i \leq n$; these sets overlap.

(P2) A finite directed labeled graph $\mathcal{E}=(G, V, \mathcal{E})$ with vertex set $V$ consisting of the integers $1,2 \ldots, n$, with $\mathcal{E} \subset V \times V \times \mathcal{A}$, with each labeled edge assigned data $\left(i(e), f(e), j_{e}\right)$ in which $i(e), f(e) \in V$ denote the initial and final vertices of the directed edge, and the label $j_{e} \in \mathcal{A}=\{0,1, \ldots, p-1\}$ is drawn from the usual alphabet of $p$-adic digits. No two edges have the same data $(i, f, j)$. Each vertex of the underlying directed graph $G$ has at least one exit edge.

(P3) To the label $j_{e}$ is associated a $p$-adic similarity map

$$
\phi: \mathbb{Z}_{p} \longrightarrow \mathbb{Z}_{p}
$$

given by

$$
\phi_{e}(y)=p y+j_{e} .
$$

This is a contractive mapping in the $p$-adic metric.

Note that in this construction the similarity maps $\phi_{e}$ in (P3) will be applied to sets in the direction reverse to that assigned to the directed graph edge $e$ of $G$, compare (2.6) below.

This definition differs from the Mauldin and Williams real number graph-directed construction in several ways. Firstly, in condition (P1) it starts with initial sets $J_{i}$ that have overlaps, which is forbidden in (G1) of the Mauldin-Williams construction. Secondly, in condition (P2) the underlying directed graph $G$ (ignoring labels) is permitted to have loops $(i(e)=t(e))$ and multiple edges (having same $i(e), v(e)$ ), which are forbidden in (G2). (Mauldin-Williams forbid these conditions in order to handle maps having different contraction ratios $t_{i, j}$ on different edges.) Thirdly, condition (P3) requires that all contraction ratios $t_{i, j}$ be equal, which is a narrower condition than the Mauldin-Williams condition (G3). We note that Condition (P3) implies that analogues of conditions (G3) (a), (b) automatically hold, aside from the non-overlapping condition:

(a) the initial sets $J_{j}=\mathbb{Z}_{p}$ satisfy the condition

$$
J_{j} \supseteq \bigcup\left\{\phi_{e}\left(J_{f(e)}\right): e=(i(e), f(e)) \text { has initial vertex } i(e)=j\right\} \text {; }
$$

(b) each map $\phi_{e}$ has $p$-adic contraction ratio

$$
t_{e} \stackrel{\text { def }}{=}|p|_{p}=\frac{1}{p}<1 .
$$


Thus for $\left[e_{1}, \ldots, e_{q}, e_{q+1}=e_{1}\right]$ a directed cycle of edges in $G$, the contracting cycle condition holds:

$$
\prod_{j=1}^{q} t_{i}\left(e_{j}\right), f\left(e_{j}\right)<1,
$$

The following result gives existence and uniqueness for the $p$-adic construction.

Theorem 2.6 ( $p$-adic geometric graph-directed construction). Let $\mathscr{G}=(G, V, \mathcal{E})$ be a connected labeled graph with vertices $V=\{1,2, \ldots, n\}$, and with edge label alphabet $\mathcal{A}=\{0,1,2, \ldots, p-1\}$. Then there exist unique nonempty compact sets $\left\{K_{i}: i \in V\right\}$, each contained in $\mathbb{Z}_{p}$, that satisfy the set-valued functional relations, for each vertex $i \in V$,

$$
K_{i}=\bigcup_{\{e: i(e)=i\}} \phi_{e}\left(K_{f(e)}\right)
$$

Proof. The existence and uniqueness of the compact set-valued fixed point (2.6) follow from Hutchinson [12], Theorem 3.1. The Hutchinson proof establishes that the $K_{i}$ are obtained by the following iterative process. We start with initial sets $K_{i}^{(0)}=\mathbb{Z}_{p}$, and iteratively define, for each vertex $i \in V$,

$$
K_{i}^{(k+1)} \stackrel{\text { def }}{=} \bigcup_{\{e: i(e)=i\}} \phi_{e}\left(K_{f(e)}^{(k)}\right)
$$

We obtain a sequence of closed sets

$$
K_{i}^{(0)} \supseteq K_{i}^{(1)} \supseteq K_{i}^{(2)} \supset \ldots,
$$

and these converge downwards to the compact sets $K_{i}$, as $n \rightarrow \infty$.

Definition 2.7. Any set $Y \stackrel{\text { def }}{=} K_{i}$ for some sub-object $K_{i} \subseteq \mathbb{Z}_{p}$ in a (restricted) $p$-adic graph directed system is called a geometric p-adic path set fractal. We denote the set of all such $Y \subseteq \mathbb{Z}_{p}$ as $\mathcal{C}_{G}\left(\mathbb{Z}_{p}\right)$.

In Theorem 2.10 below we will show that this definition gives exactly the same class of sets as those defined in the introduction, i.e. $\mathcal{C}_{G}\left(\mathbb{Z}_{p}\right)=\mathscr{\ell}\left(\mathbb{Z}_{p}\right)$.

Many different construction pairs $(\mathcal{E}, v)$ can produce the same geometric $p$-adic path set fractal $Y$. One can use this freedom to make good choices for $\mathscr{E}$. For example, one may require $\mathcal{G}$ to be right-resolving, and in addition to have at most one directed edge between any directed pair of vertices, see the proof of Theorem 3.1(1) in Section 3. 
2.3. Path sets and $\boldsymbol{p}$-adic path set fractals. We now show that geometric $p$-adic path set fractals in Section 2.2 comprise exactly the same sets as the images in $\mathbb{Z}_{p}$ of abstract path sets under a symbol labeling. We recall a formal definition of path set given in [2]. Let $\mathcal{A}^{\mathbb{N}}$ be the full one-sided shift space on $\mathcal{A}$. A pointed graph over an alphabet $\mathcal{A}$ consists of a pair $(\mathscr{E}, v)$, where $\mathscr{G}=(G, \mathcal{E})$ is a finite edge-labeled directed graph $G$, with labeled edges $\mathcal{E} \subset E \times \mathcal{A}$ having labels drawn from an alphabet $\mathcal{A}$, and $v$ a vertex of $G$. We let $V(\mathscr{E})$ and $E(\mathscr{G})$ denote the set of vertices and directed edges of $G$, respectively. Following [2] we make a basic definition.

Definition 2.8. For a pointed graph $(\mathcal{G}, v)$ its associated (abstract) path set (or pointed follower set) $\mathcal{P}=X \mathscr{E}(v) \subset \mathcal{A}^{\mathbb{N}}$ is the set of all infinite one-sided symbol sequences giving the successive labels of all one-sided infinite walks in $\mathcal{G}$ issuing from the distinguished vertex $v$. Many different $(\mathcal{G}, v)$ may give the same path set $\mathcal{P}$, and we call any such $(\mathscr{G}, v)$ a presentation of $\mathcal{P}$.

Recall that the class $\mathscr{C}\left(\mathbb{Z}_{p}\right)$ of $p$-adic path set fractals consists of images $f_{p}(\mathcal{P})=$ $f_{p}(X \mathscr{G}(v))$ of a path set $\mathcal{P}$ under a digit assignment map $\bar{f}_{p}$, sending a path address to a $p$-adic expansion. We show that the class $\mathcal{C}\left(\mathbb{Z}_{p}\right)$ agrees with the geometric class $\mathcal{C}_{G}\left(\mathbb{Z}_{p}\right)$ given by the geometric Mauldin-Williams construction. For this purpose it is helpful to know that every element of $\mathcal{C}\left(\mathbb{Z}_{p}\right)$ has presentation $Y \stackrel{\text { def }}{=}\left(\bar{f}_{p}, \mathscr{E}, v\right)$ of the special form called a standard presentation in Section 1.1.

Proposition 2.9 (Standard presentation). (1) Every path set $\mathcal{P}=X_{\mathscr{E}}(v)$ on an alphabet $\mathcal{A}$ has a presentation $(\mathcal{G}, v)$ that is right-resolving and reachable.

(2) Every p-adic path set fractal $Y$ in $\mathcal{C}\left(\mathbb{Z}_{p}\right)$ has a presentation $Y=\left(\bar{f}_{p}, \mathcal{E}, v\right)$ that is injective, right-resolving and reachable; that is, a standard presentation. Furthermore one may specify that the presentation alphabet is $\mathcal{A}=\{0,1, \ldots, p-1\}$ with the identity digit assignment map $\bar{l}_{p}$.

Proof. (1) Any path set $X$ on any alphabet $\mathcal{A}$ has a right-resolving, reachable presentation $\mathcal{P}=(\mathscr{E}, v)$ on this alphabet, with $X=X_{\mathscr{E}}(v)$, by a standard construction, see [2], Theorem 3.2.

(2) By hypothesis $Y \in \mathcal{C}\left(\mathbb{Z}_{p}\right)$ comes with a presentation $Y=f_{p}\left(X_{\mathscr{G}^{\prime}}\left(v^{\prime}\right)\right)$, on an alphabet $\mathcal{A}^{\prime}$. Using the digit assignment map $\bar{f}_{p}: \mathcal{A}^{\prime} \rightarrow\{0,1, \ldots, p-1\}$, we may relabel the underlying edges of the graph of $\mathscr{E}^{\prime}$ by the image labels in $\mathcal{A}=$ $\{0,1, \ldots, p-1\}$, obtaining a labeled directed graph $\mathcal{G}^{\prime \prime}$ with $Y=i_{p}\left(X_{\mathscr{G}^{\prime \prime}}\left(v^{\prime}\right)\right)$. By (1) the path set $X_{\mathscr{G}^{\prime \prime}}(v)$ has another presentation $X \mathscr{E}(v)$, in which the new graph $\mathscr{E}$ is right-resolving and reachable, and uses the same label alphabet $\mathcal{A}$. By inspection $Y \stackrel{\text { def }}{=}\left(\bar{l}_{p}, \mathcal{E}, v^{\prime}\right)$ is still a presentation of $Y$, and it is injective, right-resolving and reachable. 
Note the one-sided shift space $\mathcal{A}^{\mathbb{N}}$, for $\mathcal{A}=\{0,1, \ldots, p-1\}$, topologized with the product topology, is homeomorphic to $\mathbb{Z}_{p}$ with its usual $p$-adic topology, where one identifies a symbol sequence $\alpha_{0} \alpha_{1} \alpha_{2} \cdots \in \mathcal{A}^{\mathbb{N}}$ with the $p$-adic expansion

$$
x=\sum_{j=0}^{\infty} \alpha_{j} p^{j}=\left(\ldots \alpha_{2} \alpha_{1} \alpha_{0}\right)_{p} \in \mathbb{Z}_{p} .
$$

This is exactly the map $i_{p}$ underlying the standard presentation $Y=\left(\bar{i}_{p}, \mathcal{E}, v\right)$ of a $p$-adic path set fractal above.

Now we relate the class $\mathcal{C}\left(\mathbb{Z}_{p}\right)$ to the geometric class $\mathcal{C}_{G}\left(\mathbb{Z}_{p}\right)$. To this end we note that attached to any labeled directed graph $\mathcal{E}=(G, V, \mathcal{E})$ on alphabet $\mathcal{A}=$ $\{0,1, \ldots, p-1\}$ there is associated a (restricted) $p$-adic graph-directed construction, (as in Section 2.2) based on the same graph data $\mathcal{E}=(G, V, \mathcal{E})$ where the edge label $j \in \mathcal{A}$ is now assigned the map $\phi_{j}(x)=p x+j$.

Theorem 2.10. There holds $\mathcal{C}_{G}\left(\mathbb{Z}_{p}\right)=\mathscr{C}\left(\mathbb{Z}_{p}\right)$. Specifically, we have what follows.

(1) Let $K_{v} \in \mathscr{\ell}_{G}\left(\mathbb{Z}_{p}\right)$ be a construction sub-object of a (restricted) p-adic graph directed construction with data $\mathcal{E}=(G, V, \mathcal{E})$ using edge maps $\phi_{e}=p x+j_{e}$ with $0 \leq j_{e} \leq p-1$, associated to vertex $v$ of $G$. Create a path set from the same data $\mathcal{E}(G, V, \mathcal{E})$, interpreting the edge labels $j_{e} \in \mathcal{A}=\{0,1, \ldots, p-1\}$, and let $Y \stackrel{\text { def }}{=} i_{p}(X \mathscr{E}(v))$ be the $p$-adic path set fractal with presentation $Y \stackrel{\text { def }}{=}\left(\bar{\imath}_{p}, \mathscr{E}, v\right)$. Then $Y=K_{v}$. It follows that $\mathcal{C}_{G}\left(\mathbb{Z}_{p}\right) \subset \mathcal{C}\left(\mathbb{Z}_{p}\right)$.

(2) Let $Y \in \mathscr{C}\left(\mathbb{Z}_{p}\right)$ be any $p$-adic path set fractal. Then it has a standard presentation $Y=\left(\bar{i}_{p}, \mathcal{E}, v\right)$ with a labeled directed graph $\mathcal{E}=(G, V, \mathcal{E})$, having the additional property that all vertices of the graph $G$ have at least one exit edge. By (1) there is an associated (restricted) p-adic graph directed construction having a sub-object $K_{v}$, with $Y=K_{v}$. Thus $\mathscr{C}\left(\mathbb{Z}_{p}\right) \subset \mathscr{C}_{G}\left(\mathbb{Z}_{p}\right)$.

Proof. (1) The correspondence between $K_{v}$ and $Y$ proceeds by relating paths to the address labels of points in the graph-directed fractal, compare Edgar [7], Section 4.3. We study the set-valued iteration $K_{i}^{(k)}$ given in Theorem 2.6 for the geometric $p$-adic path set fractal determined by $\mathscr{G}=(G, V, \mathcal{E})$. We prove by induction on $k \geq 0$ that for all vertices $i$,

$$
K_{i}^{(k)} \stackrel{\text { def }}{=} \bigcup\left(\left(\alpha_{0}+\alpha_{1} p+\cdots+\alpha_{k-1} p^{k-1}\right)+p^{k} \mathbb{Z}_{p}\right),
$$

where the set union is taken over label sequences $\left(\alpha_{0}, \ldots, \alpha_{k-1}\right)$ of legal walks in the directed graph $\mathcal{E}$ of length $k$ starting from vertex $i$. (The edge leaving the initial vertex $i$ has label $\alpha_{0}$.) The hypothesis that an exit edge exists from each vertex guarantees that all paths extend one step. The base case $k=0$ holds since all $K_{i}^{(0)}=\mathbb{Z}_{p}$. For the induction step, we have that $K_{i}^{(k+1)}$ is comprised of sets

$$
\phi_{e}\left(K_{f(e)}^{(k)}\right)=j_{e}+\sum_{i=0}^{k-1} \alpha_{i} p^{i+1}+p^{k+1} \mathbb{Z}_{p},
$$


where $\left(\alpha_{0}, \ldots, \alpha_{k-1}\right)$ are labels from a directed walk in $\mathcal{E}$ starting from vertex $f(e)$. But now $\left(j_{e}, \alpha_{0}, \ldots, \alpha_{k-1}\right)$ are vertices of a directed walk of length $k+1$ starting from vertex $i$, and all such walks are enumerated this way. This completes the induction step. Letting $k \rightarrow \infty$, for each vertex $i$ these sets decrease to $K_{i}$, which is now identified with all infinite walks in $\mathcal{E}$ starting from vertex $i$. Choosing $i$ to be the original marked vertex, we obtain $K_{i}=\bar{l}_{p}(X \mathscr{E}(v))=Y_{v}$, as asserted.

(2) Given a $p$-adic path set fractal $Y$ we take a standard form presentation $Y=$ $\left(\bar{\imath}_{p}, \mathcal{G}^{\prime}, v\right)$. We now prune the graph $\mathscr{G}^{\prime}$ to remove any vertices with no exit edges, since such vertices contribute no infinite paths to the path set $\mathcal{P}=X_{\mathscr{E}}(v)$, and leave $Y$ unchanged. The new graph may still have vertices with no exit edges, but by repeating this operation a finite number of times, we will arrive at a presentation $Y=\left(\bar{l}_{p}, \mathcal{G}, v\right)$ in which all vertices have at least one exit edge. The right-resolving and reachability properties are unaffected, so the new presentation is still standard. The construction of part (1) now applies to give the result.

It is known that the class of path sets on a fixed alphabet is closed under finite unions and intersections ( [2], Theorem 1.1). Theorem 2.10 implies that the collection of $p$-adic path set fractals $\mathcal{C}\left(\mathbb{Z}_{p}\right)$ is closed under set union and intersection as well.

2.4. One element $p$-adic path set fractals. We characterize path sets consisting of a single element.

Theorem 2.11 (Single element $p$-adic path set fractals). The $p$-adic path set fractals $Y \in \mathcal{C}\left(\mathbb{Z}_{p}\right)$ that consist of a single element are exactly those $Y=\{r\}$ for which $r$ is a p-integral rational number, i.e. $r \in \mathbb{Q} \cap \mathbb{Z}_{p}$.

Remark 2.12. This simple result supplies a dynamical characterization of the $p$-integral rational numbers $r$ inside $\mathbb{Z}_{p}$.

Proof. Given a $p$-adic path set fractal $Y$, assume it is given with a standard presentation $\left(\bar{i}_{p}, \mathscr{g}, v_{0}\right)$. Using the pruning construction used in proving Theorem 2.10 we may without loss of generality assume each vertex in $\mathscr{E}$ has at least one exit edge. Such a presentation has an underlying path set $X_{\mathscr{E}}\left(v_{0}\right)$ consisting of a single infinite path if and only if there is exactly one exit edge from each vertex, and if the path is eventually periodic. The latter forces any element $r$ to be a rational number in $\mathbb{Z}_{p}$. Conversely, we may easily construct a path set consisting of a single element giving the $p$-adic expansion of $r$.

\section{Hausdorff dimension of $p$-adic path set fractals}

We obtain a formula for the Hausdorff dimension of a $p$-adic path set fractal $Y$, computable from a standard form presentation of $Y$. The formula is based on a 
Hausdorff dimension relation between $p$-adic path set fractals and graph-directed constructions on the real numbers.

To state the result, we note that the adjacency matrix $A=A(G)$ of a directed graph $G$ is a non-negative integer matrix whose rows and columns are numbered by the vertices of $G$ (in the same order) with entry $A_{i j}$ counting the number of directed edges outgoing from vertex $i$ and incoming to vertex $j$.

Theorem 3.1 (Hausdorff dimension formula). Let $Y$ belong to $\mathcal{C}\left(\mathbb{Z}_{p}\right)$, and suppose that $Y \stackrel{\text { def }}{=}\left(\bar{f}_{p}, \mathcal{E}, v\right)$ is a standard form presentation of $Y$.

(1) The map $\iota_{p}: \mathbb{Z}_{p} \rightarrow[0,1] \subset \mathbb{R}$ sending $\alpha=\sum_{k=0}^{\infty} \alpha_{k} p^{k} \in \mathbb{Z}_{p}$ to the corresponding real number with base $p$ radix expansion

$$
\iota_{p}(\alpha) \stackrel{\text { def }}{=} \sum_{k=0}^{\infty} \frac{\alpha_{k}}{p^{k+1}}
$$

is a continuous map. Under this map the image set $K_{0} \stackrel{\text { def }}{=} \iota_{p}(Y) \subset[0,1]$ is a construction sub-object of a Mauldin-Williams graph-directed fractal whose edge similarity maps are all contracting similarity maps of $\mathbb{R}$ with contraction ratio $1 / p$.

(2) The Hausdorff dimensions of these sets are related by

$$
d_{H}(Y)=d_{H}\left(K_{0}\right) .
$$

(3) The Hausdorff dimension

$$
d_{H}(Y)=d_{H}\left(K_{0}\right)=\log _{p} \alpha,
$$

where $\alpha=\sigma(A(\mathscr{E}))$ is the spectral radius of the adjacency matrix $A=A(\mathscr{E})$ of the underlying directed graph $G$ of $\mathcal{E}$.

Remarks 3.2. (1) In Theorem 1.13 of [2] the topological entropy of a path set $X=X \mathscr{g}(v)$, with a right-resolving reachable presentation is given by

$$
h_{\text {top }}(X)=\log \sigma(A(\mathscr{G})),
$$

where $\sigma(A(\mathscr{E}))$ is the spectral radius as above. We deduce from (3) above that for any $p$-adic path set fractal $Y$ constructed from $X$ by an injective presentation $Y \stackrel{\text { def }}{=}\left(\bar{f}_{p}, \mathcal{E}, v\right)$, its Hausdorff dimension is

$$
d_{H}(Y)=\frac{h_{\text {top }}(X)}{\log p} .
$$

This extends to path sets, a result that is well known in the shift-invariant case (Furstenberg [11], Proposition III.1). 
(2) The standard presentation assumption for $Y$ above is needed to guarantee equality of the Hausdorff dimension with $\log _{p} \alpha$. For a general presentation $Y \stackrel{\text { def }}{=}$ $\left(\bar{f}_{p}, \mathcal{E}, v_{0}\right)$ of $X$ the adjacency matrix counts the growth rate of number of paths, which upper bounds the number of distinct sequences of path labels. That is, one always has

$$
d_{H}(Y) \leq \frac{h_{\text {top }}(X \mathscr{E}(v))}{\log p} \leq \log _{p} \sigma(A(\mathscr{E})) .
$$

(3) The allowed values $\sigma(A(\mathscr{E}))$ that may occur above are exactly the class of positive real algebraic integers called Perron numbers, introduced by Lind [15].

Proof. (1) The map $\iota_{p}: \mathbb{Z}_{p} \rightarrow[0,1]$ is surjective and one-to-one away from a countable set. It is continuous because the $p$-adic topology is strictly finer than the comparable topology on base $p$ expansions of real numbers.

We are given a standard presentation of $Y=\left(\bar{l}_{p}, \mathscr{g}, v\right)$, where without loss of generality the alphabet $\mathcal{A}=\{0,1, \ldots, p-1\}$. The graph $\mathscr{G}$ is right-resolving and reachable, but this will not be sufficient to obtain a Mauldin-Williams construction for the image of $X_{\mathscr{E}}(v)$ preserving symbol sequences. We need a standard presentation with extra properties. We call a presentation right-separating if the underlying directed graph $G$ of $\mathscr{E}$ has no multiple edges.

Claim. There exists a presentation $Y=\left(\bar{\imath}_{p}, \mathcal{E}, v\right)$ in which $\mathcal{E}$ is both rightresolving and right-separating.

To show the claim, given a right-resolving presentation $\left(\mathscr{E}^{\prime}, v^{\prime}\right)$, we show it may be converted to a right-separating presentation by making use of a vertex-splitting construction, as follows. Suppose that a vertex $v$ of $\mathscr{\ell}^{\prime}$ has $k \geq 2$ labeled edges from a vertex $w$, which necessarily has distinct labels. We create a new labeled graph $\mathscr{E}^{\prime \prime}$ that retains all vertices of $\mathcal{E}$ except $v$ and replaces $v$ by $k$ vertices $v^{(i)}, 1 \leq i \leq k$.

Case 1: $w \neq v$. In this case we assign a single new labeled edge from $w$ to each $v^{(i)}$ such that as $i$ varies, the corresponding edge labels exhaust the $k$ labels of edges from $w$ to $v$. The exit edges assigned each $v^{(i)}$ each duplicate the exit edges from $v$, both in multiplicity and in labels, with self-loops of $v$ corresponding to self-loops of $v^{(i)}$. The entering edges to $v^{(1)}$ will all be the same in multiplicity and in labels as for $v$, while for $v^{(i)}$ with $i \geq 2$ there will be no entering edges from the rest of the graph, with the exception of self-loops, assigned as above. Finally all edges between any two vertices distinct from the $v^{(i)}$ will be the same as in the original graph.

Case 2: $\boldsymbol{w}=\boldsymbol{v}$. In this case $v$ has $k$ self-loops, which have distinct labels since the right-resolving property is assumed. We may identify the $k$ loop labels with $\{1,2, \ldots, k\}$ in some fixed fashion, and then assign a directed edge from $v^{(i)}$ to $v^{(j)}$ with edge label corresponding to $i+j(\bmod k)$, for $1 \leq i, j \leq k$. The other exit edges assigned each $v^{(i)}$ will duplicate the exit edges of $v$, in multiplicity and labels (excluding self-loops). The entering edges of $v^{(1)}$ will be the same in multiplicity and labels as for $v$ (excluding self-loops). All other $v^{(i)}$ have no entering edges coming from any other vertices of the original graph $v^{\prime} \neq v$. 
Now that $\mathscr{G}^{\prime \prime}$ is constructed, we assert that the path sets from all states $\left(\mathscr{G}^{\prime \prime}, w^{\prime}\right)$ for $w^{\prime} \neq v^{(i)}$ agree with those of $\left(\mathscr{E}^{\prime}, w^{\prime}\right)$, while all path sets $\left(\mathscr{E}^{\prime \prime}, v^{(i)}\right)$ agree identically with that of $\left(\mathscr{\ell}^{\prime}, v\right)$. This assertion may be established by viewing $\mathscr{\ell}^{\prime}$ as a covering of $\mathcal{G}$ which preserves edge labels, which has the $k$ vertices $v^{(i)}$ lying above vertex $v$, and all other vertices agreeing. One may check that each edge in $\mathscr{\ell}^{\prime}$ from a given initial vertex $v^{\prime}$ lifts uniquely to a suitable vertex and edge above it in $\mathscr{\ell}^{\prime \prime}$ (here $v^{\prime}=w$ is the only interesting case), except that self-loops from $v$ lift to a self-loop for any initial vertex $v^{(i)}$. After the first step, any path lifts uniquely to $\mathcal{E}^{\prime \prime}$. Conversely any labeled path in the lifted graph projects to an allowable labeled path in $\mathscr{G}^{\prime}$. The assertion follows.

This construction has the feature that the new graph $\mathcal{E}^{\prime \prime}$ is still right-resolving. In consequence the construction may be repeated. In doing so, we must eventually arrive at a right-resolving presentation that is also right-separating. To see this, assign to each vertex an integer invariant that is the product of the multiplicities of all entering edges. When a vertex is split, this invariant decreases for all of the $k$ descendants $v^{(i)}$, and remains the same for all other vertices of the graph. By the well-ordering of $\mathbb{N}$, the splitting procedure will eventually halt at a right-separating presentation. This establishes the claim.

We have now obtained a standard presentation that is also a right-separating presentation $\left(\bar{l}_{p}, \mathcal{G}, v\right)$ of $Y$. By pruning vertices with no exit edges (repeating the operation finitely many times, as necessary), we may obtain such a presentation in which additionally each vertex has at least one exit edge. Associated to $\mathscr{G}$ are $|V(\mathscr{G})|$ path sets $X_{\mathscr{E}}(\boldsymbol{w}), \boldsymbol{w} \in V(\mathscr{E})$, and corresponding $Y_{\boldsymbol{w}} \stackrel{\text { def }}{=} i_{p}\left(X_{\mathscr{E}}(\boldsymbol{w})\right) \in \mathcal{C}\left(\mathbb{Z}_{p}\right)$.

We now proceed to map these sets to real image sets which are corresponding graph-directed constructions. For convenience we re-number the vertices of $\mathcal{G}, 0 \leq$ $i \leq n$, where $n=|V(\mathscr{G})|-1$, with vertex 0 corresponding to the original $v$. We map the individual sets $Y_{j}$ under the map $\iota_{p}+2 j$ to the image sets

$$
K_{j} \stackrel{\text { def }}{=} \iota_{p}\left(Y_{j}\right)+2 j \subset[2 j, 2 j+1] .
$$

The integer shifts by $2 j$ make all sets $K_{j}$ disjoint in $\mathbb{R}$; Thus $K_{0}=\iota(Y)$.

We show below that the sets $\left(K_{0}, K_{1}, \ldots, K_{n}\right)$ are the complete set of construction sub-objects of a particular Mauldin-Williams graph-directed construction. The integer shifts made above enforce the non-overlapping condition needed in that construction.

We set up a Mauldin-Williams geometric graph-directed construction in $\mathbb{R}$, which has construction object $K$ contained in the compact set $[0,2 n]$, for which the sets $\left(K_{0}, K_{1}, \ldots, K_{n}\right)$ will form the construction sub-objects. The initial sets will be $J_{j}=[2 j, 2 j+1]$ for $0 \leq j \leq n$; they satisfy the non-overlapping property (G1). It uses the same directed graph $G$ as that of $\mathcal{E}$. The graph $G$ has all the correct properties (G2) to be a graph in the Mauldin-Williams construction: it is connected, has at most one directed edge between any ordered pair of vertices (by the rightseparating property), and each vertex has at least one exit edge. To each directed 
labeled edge $e=\left[i_{1}, i_{2}\right]$ of $G$ with label $j_{e}$ and map $\phi_{e}$ we associate the real-valued map

$$
T_{e}(x)=T_{i_{1}, i_{2}}(x) \stackrel{\text { def }}{=} \frac{1}{p}\left(\left(x-2 i_{2}\right)+j\right)+2 i_{1} .
$$

This map is a similarity with contraction ratio $1 / p$, and note that

$$
T_{i_{1}, i_{2}}\left(J_{i_{2}}\right) \subset J_{i_{1}} .
$$

Now condition (G3)(a), that the sets $\left\{T_{i, j}\left(J_{j}\right): e=(i, j)\right\}$ are non-overlapping for each $i$, holds as a consequence of the right-resolving property of $\mathcal{E}$. The second condition

$$
J_{i} \supseteq \bigcup\left\{T_{i, j}\left(J_{j}\right) \mid(i, j) \in G^{*}\right\}
$$

follows from (3.2). Finally condition (G3)(b) holds since every map $T_{e}(x)$ is a strict contraction.

By the basic theorem of Hutchinson [12], Theorem 3.1, this construction has a unique compact attractor $K$ consisting of a collection of disjoint compact sub-objects $\left\{K_{j}: 0 \leq j \leq n\right\}$, and it remains to verify that

$$
K_{0}=\iota_{p}(Y) .
$$

The sets $K_{j}$ are obtained by the Mauldin-Williams (iterative) geometric graphdirected construction using [20], Theorem 1, starting with the (disjoint) initial sets $J_{j} \stackrel{\text { def }}{=}[2 j, 2 j+1]$. After $m$-iterations, we have sets $J_{j}^{(k)}$ which for fixed $j$ form nested sequences of compact sets, each having nonempty interior. We then obtain the limit sub-objects $K_{j} \stackrel{\text { def }}{=} \bigcap_{k} J_{j}^{(k)}$. The Mauldin-Williams construction object is $K=\cup_{0 \leq j \leq n} K_{j}$. One can prove by induction on $k$ that

$$
J_{i}^{(k)}=2 j+\bigcup\left(\frac{\alpha_{0}}{p}+\frac{\alpha_{1}}{p^{2}}+\cdots+\frac{\alpha_{k-1}}{p^{k}}+\frac{1}{p^{k}}[0,1]\right),
$$

where the set union runs over all symbol sequences of length $k$ on the labeled directed graph $\mathcal{E}$ starting from vertex $i$. From this construction one sees that $K_{0}=\iota_{p}(Y)$ since $K_{0} \subset[0,1]$ and the underlying symbol sequences of $Y$ and of $K_{0}$ agree. Moreover one sees that $K_{j} \subset[2 j, 2 j+1]$ and all the construction sub-objects satisfy $K_{j}=\iota_{p}\left(Y_{j}\right)+2 j, 0 \leq j \leq n$.

(2) The definition of $p$-adic Hausdorff dimension is quite similar to Hausdorff dimension for real numbers on the interval [0, 1], cf. Abercrombie [1]. An $\epsilon$-covering of $Y \subset \mathbb{Z}_{p}$ is a covering of $Y$ by a countable collection of $p$-adic open balls all having diameter at most $\epsilon$. Now consider the quantities

$$
m_{\beta}(Y) \stackrel{\text { def }}{=} \lim _{\epsilon \rightarrow 0}\left(\inf _{\epsilon \text {-cover }}\left(\operatorname{Vol}\left(B\left(x_{i}, \epsilon_{i}\right)\right)\right)^{\beta}\right),
$$

in which the data $\left\{\left(x_{i}, \epsilon_{i}\right): i \geq 1\right\}$ describes the covering, specifying center $x_{i}$ and radius $\epsilon_{i}$ of $p$-adic disks, with all $0<\epsilon_{i} \leq \epsilon$, and $\operatorname{Vol}(S)$ denotes the usual $p$-adic 
measure of $S \subset \mathbb{Z}_{p}$. There is a cutoff value $\beta_{0}$ such that $m_{\beta}(Y)=0$ for $\beta>\beta_{0}$ and $m_{\beta}(Y)=\infty$ for $\beta<\beta_{0}$; this is the Hausdorff dimension of $Y$. We use the following basic fact, following [13], Section 3.2.

Claim. The mapping $\iota_{p}: \mathbb{Z}_{p} \rightarrow[0,1]$ which sends a $p$-adic number $\lambda=$ $\left(\ldots \alpha_{2} \alpha_{1} \alpha_{0}\right)_{p}$ to the real number with base $p$ expansion $\alpha_{0} \alpha_{1} \alpha_{2} \ldots$ is continuous and one-to-one off a countable set. This mapping preserves Hausdorff dimension, i.e $d_{H}(Y)=d_{H}\left(\iota_{p}(Y)\right)$.

To verify the claim, note that one can expand each set in a $p$-adic covering of a set $Y$ to a closed-open disk

$$
B\left(m, p^{j}\right)=\left\{x \in \mathbb{Z}_{p}: x \equiv m\left(\bmod p^{j}\right)\right\}
$$

(which has diameter $\frac{1}{p^{j}}$ ), with at most a factor of $p$ increase in diameter, and similarly one can inflate any real covering to a covering with ternary intervals $\left[\frac{m}{p^{j}}, \frac{m+1}{p^{j}}\right]$ with at most a factor of $p$ increase in diameter. But these special intervals are assigned the same diameter under their respective metrics, and this can be used to show the Hausdorff dimensions of $Y$ and $\iota_{p}(Y)$ coincide. (The Hausdorff measures of the resulting sets are not proved to coincide by this argument.)

The truth of the claim immediately yields $d_{H}\left(K_{0}\right) \equiv d_{H}\left(\iota_{p}(Y)\right)=d_{H}(Y)$, as asserted.

(3) We are given a standard presentation $Y=\left(\bar{\imath}_{p}, \mathscr{E}, v_{0}\right)$ of the $p$-adic path set fractal $Y$.

Assume first that this presentation is right-separating. In that case we can directly apply the formulas of Mauldin-Williams to the construction made in (1). The set $K_{v_{0}}$ is connected by directed paths to every vertex of the graph $\mathscr{G}$, so that the vertex set $C_{1}=S C(\mathscr{G})$ consists of all strongly connected components of $\mathscr{G}$. By Proposition 2.4 the Hausdorff dimension of the sub-object $K_{v_{0}}$ is then the same as that of the full construction object $K=\bigcup_{v} K_{v}$.

The Hausdorff dimension of the full object $K$ can now be computed using Proposition 2.3. In our case all nonzero maps for $G$ are similarities with constant ratio $1 / p$, which yields the formula for the scaled construction matrix

$$
A_{\beta}=\left[t_{i, j}^{\beta}\right]_{1 \leq i, j \leq n}=\left(\frac{1}{p}\right)^{\beta} A_{G},
$$

in which $A_{G}$ is the adjacency matrix of the directed graph $G$, given by

$$
A_{G}=\left[m_{i, j}\right]_{1 \leq i, j \leq n}
$$

where $m_{i, j}$ counts the number of directed edges from vertex $v$ to vertex $w$. Now set

$$
\Phi(\beta) \stackrel{\text { def }}{=} \text { Spectral radius of } A_{\beta},
$$

and the special form of $A_{\beta}$ yields

$$
\Phi(\beta)=\lambda_{0} p^{-\beta},
$$


in which $\lambda_{0}$ is the spectral radius $\sigma\left(A_{G}\right)$. By Proposition 2.3 the full construction object has Hausdorff dimension

$$
d_{H}(K)=\alpha
$$

where $\Phi(\alpha)=1$. This requires

$$
\alpha=\log _{p} \lambda_{0}=\log _{p} \sigma\left(A_{G}\right)
$$

the desired formula.

It remains to treat the general case, in which the initial standard presentation $Y=\left(\bar{l}_{p}, \mathcal{E}, v_{0}\right)$ is not necessarily right-separating. We show that the formula for Hausdorff dimension continues to hold. To handle this case, we study the effect of the state-splitting construction introduced earlier to convert a right-resolving presentation $\left(\mathscr{G}^{\prime}, v^{\prime}\right)$ of a path set to a presentation $\left(\mathscr{E}, v_{0}\right)$ that is also right-separating. It suffices to show that every step of this procedure yielding a graph $\left(\mathcal{E}^{\prime \prime}, v^{\prime}\right)$ preserves the value of the spectral radius (Perron eigenvalue) of the associated nonnegative integer matrix $A \mathscr{E}^{\prime \prime}$. If this is shown, then the already proved formula for the Hausdorff dimension for the $p$-adic path set associated to $\left(\mathcal{E}, v_{0}\right)$ will carry over to that for $\left(\mathscr{E}^{\prime}, v^{\prime}\right)$.

To check that the spectral radius is preserved under this operation, we use the known fact that the spectral radius of a nonnegative matrix $A$ is given by

$$
\sigma(A)=\lim _{k \rightarrow \infty}\left(N_{k}(A)\right)^{1 / k}
$$

in which $N_{k}(A)=\boldsymbol{e}^{T} A^{k} \boldsymbol{e}$, where $\boldsymbol{e}=[1,1, . ., 1]^{T}$ is a column vector. Here $N_{k}(A)$ counts the number of directed paths of length $k$ between all pairs of vertices of $A$. (The existence of the limit is part of the assertion.) We use the fact that $\mathcal{E}^{\prime \prime}$ is a covering of $\mathscr{G}^{\prime}$ and that all (labeled) paths of $\boldsymbol{G}^{\prime}$ lift uniquely to paths of $\mathscr{G}^{\prime \prime}$, with the exception of paths that have starting vertex $v$, which have $s$ distinct lifts, where $s$ was the number of vertices of $\mathcal{G}$ that were split. From this we conclude that

$$
N_{k}\left(A_{G^{\prime}}\right) \leq N_{k}\left(A_{G^{\prime \prime}}\right) \leq s N_{k}\left(A_{G^{\prime}}\right) .
$$

Since $s$ is constant, we conclude that

$$
\sigma\left(A_{G^{\prime}}\right) \leq \sigma\left(A_{G^{\prime \prime}}\right) \leq \lim _{k \rightarrow \infty} s^{1 / k} N_{k}\left(A_{G^{\prime}}\right)^{1 / k}=\sigma\left(A_{G^{\prime}}\right)
$$

giving the result.

Proof of Theorem 1.1. This result is immediate from Theorem 3.1, combining (2) and (3).

\section{4. $p$-adic addition and path set fractals}

We analyze the effect on $p$-adic path set fractals of addition of $p$-integral rational numbers $r \in \mathbb{Q}$, viewing $\mathbb{Q}$ as a subfield of $\mathbb{Q}_{p}$. We describe algorithms which when given a presentation $\left(\mathscr{G}, v_{0}\right)$ of a path set $Y$, will produce a presentation of $Y+r$. 
4.1. Sum of a path set and a $p$-integral rational number. Theorem 1.2 is an immediate corollary of the following stronger result. Recall that a $p$-integral rational number $r$ is any $r \in \mathbb{Q} \cap \mathbb{Z}_{p}$.

Theorem 4.1. Let $Y$ belong to $\mathcal{C}\left(\mathbb{Z}_{p}\right)$, and suppose it has a standard presentation $Y \stackrel{\text { def }}{=}\left(\bar{\imath}_{p}, \mathcal{E}, v_{0}\right)$ having $V$ vertices. Suppose also that $r$ is a $p$-integral rational number, which has a p-adic expansion with pre-periodic part of length $Q_{0}$ and $a$ periodic part of period $Q$. Then the additively shifted set $Y^{\prime} \stackrel{\text { def }}{=} Y+r \in \mathcal{C}\left(\mathbb{Z}_{p}\right)$, and it has a right-resolving presentation having at most $2 p\left(Q_{0}+Q\right) V$ vertices.

Proof. We give an explicit construction of a presentation for $Y+r$ which certifies it is a $p$-adic path set fractal, starting from a given standard presentation.

Suppose first that we are in the special case where $r$ has a purely periodic $p$-adic expansion $\left(\ldots c_{2} c_{1} c_{0}\right)_{p}$, of period $Q$, with $c_{j+Q}=c_{j}$, and write

$$
r=\sum_{j=0}^{\infty} c_{j} p^{j}=\left(\sum_{j=0}^{Q-1} c_{j} p^{j}\right)\left(\sum_{k=0}^{\infty} p^{k Q}\right) .
$$

We aim to construct a standard presentation $Y^{\prime}=\left(\bar{l}_{p}, \mathscr{E}^{\prime}, \boldsymbol{w}_{0}\right)$ and show that $Y^{\prime}=Y+r$. (At the level of symbols we may identify $Y^{\prime}$ with the underlying path set $\mathcal{P}^{\prime}=\left(\mathscr{G}^{\prime}, \boldsymbol{w}_{0}\right)$ since the identity map matches them.) The vertex states of $\mathscr{G}^{\prime}$ will be labeled $\boldsymbol{w}=(v, f, e, a)$, in which:

(i) $v$ denotes a vertex of $\mathscr{E}$;

(ii) $f$ denotes a place-marker in the periodic portion of the $p$-adic expansion of $r$, and satisfies $0 \leq f \leq Q-1$;

(iii) $e$ keeps track of the current amount of carry-digit information not yet incorporated in the sum-set $p$-adic expansion;

(iv) $a$ with $0 \leq a \leq p-1$ denotes an edge label value.

The initial vertex will be $\boldsymbol{w}_{0}=\left(v_{0}, 0,0,0\right)$. We will establish the upper bound $e \leq 2$ on the maximum size of a carry-digit in the analysis below.

The exit edges of $\mathscr{G}^{\prime}$ map a vertex $\boldsymbol{w}$ to $\boldsymbol{w}^{\prime}=\left(v^{\prime}, f^{\prime}, e^{\prime}, a^{\prime}\right)$ in which there is a directed labeled edge $\left(v, v^{\prime}\right) \in \mathcal{G}$, with label $\ell_{1}$ satisfying $\ell_{1}=a^{\prime}$, and the value $f^{\prime} \equiv f+1(\bmod Q)$. The edge label $\ell^{\prime}$ assigned to this edge will be $0 \leq \ell^{\prime} \leq p-1$ with

$$
\ell^{\prime} \equiv e+\ell_{1}+c_{f}(\bmod p)
$$

and the value $e^{\prime}$ is required to satisfy

$$
e^{\prime}=\frac{1}{p}\left(e+\ell_{1}+c_{f}-\ell^{\prime}\right) .
$$

Finally we define the graph $\mathcal{E}^{\prime}$ to consist of all states reachable from the initial vertex $\boldsymbol{w}_{0}$, and all edges constructed between these states. 
We first show that all reachable vertices satisfy the carry-digit bound $e \leq 2$; this shows that the graph $\mathcal{G}^{\prime}$ is finite and also bounds its size. The carry-digit bound is proved by induction on the number of steps $n$ along a directed path. The base case $n=0$ has $e=0$. For the induction step, using the rule above, we get

$$
e^{\prime}=\frac{1}{p}\left(e+\ell_{1}+c_{f}-\ell^{\prime}\right) \leq \frac{1}{p}(2+(p-1)+(p-1)) \leq 2,
$$

completing the induction step. Therefore in this case $\mathcal{G}^{\prime}$ has at most $2 p(Q-1) V$ vertices.

We next show that the presentation $Y^{\prime}=\left(\bar{l}_{p}, \mathscr{E}^{\prime}, \boldsymbol{w}_{0}\right)$ is a standard presentation. We first check that $\mathscr{G}^{\prime}$ is right-resolving. To see this, note that the exit edges from a vertex $\boldsymbol{w}$ correspond to exit edges from vertex $v$ in the right-resolving graph $\mathscr{E}$, whence any two edges have different values of $\ell_{1}$. Now the exit edge label $\ell^{\prime}$ is an invertible linear function of $\ell_{1}$ by (4.1), since the values $e$ and $c_{f}$ are fixed by $\boldsymbol{w}$, so all exit edges have distinct labels, as asserted. This graph $\mathscr{G}^{\prime}$ is reachable from vertex $\boldsymbol{w}_{0}$ by construction, so we have a standard presentation.

We next observe that a lifted path in $\mathscr{G}^{\prime}$ uniquely determines the path in $\mathscr{E}$ it lies over. This follows since the path label value $\ell_{1}$ is uniquely recoverable from the path label $\ell^{\prime}$ and the vertex data on $\mathcal{G}$, using (4.2), since $e^{\prime}$ is known and $c_{f}$ is known from the vertex label $f$. The underlying path on $\mathscr{E}$ determines the quantity

$$
y_{n}=\sum_{k=0}^{n-1} a_{k} p^{k}, \quad i=1,2,
$$

corresponding to the initial part of the $p$-adic expansion of a value $y \in Y$ being determined by the $\mathscr{E}$-path. (The values $a_{k}$ are the successive labels $\ell_{1}$ along the $\mathcal{E}$-path.) Conversely, each path in $\mathcal{E}$ with initial vertex $v_{0}$ lifts to a unique path in $\boldsymbol{G}^{\prime}$ with initial vertex $\boldsymbol{w}_{0}$. Given a vertex $\boldsymbol{w}$, a labeled edge $\left(v, v^{\prime}\right)$ with label $\ell_{1}$ determines the values $a^{\prime}=\ell_{1}, e^{\prime}$, and a unique vertex $\boldsymbol{w}^{\prime}$ that $\boldsymbol{w}$ connects to.

We now show that for an infinite path in $\mathscr{E}$ determining $y \in Y$, the labels of the lifted path in $\mathscr{G}^{\prime}$ suffice to compute the associated value $y^{\prime} \in Y^{\prime}$. We prove this by induction on $n$, for the $n$-step initial path. The successive edge labels $\left\{\ell_{i}^{\prime}: 0 \leq i \leq\right.$ $n-1\}$ of the lifted path in $\mathcal{G}^{\prime}$ with the end vertex data $e^{\prime}=e_{n}$ determine the quantity

$$
y_{n}^{\prime} \stackrel{\text { def }}{=} \sum_{k=0}^{n-1} \ell_{k}^{\prime} p^{k}+e_{n} p^{n} .
$$

We establish by induction on $n$ that

$$
y_{n-1}^{\prime}=y_{n-1}+r_{n-1},
$$

in which

$$
r_{m} \stackrel{\text { def }}{=} \sum_{k=0}^{m-1} c_{k} p^{k}
$$


is a truncated version of the $p$-adic expansion of $r$. The base case $n=1$ is clear. By the induction hypothesis, we have

$$
\begin{aligned}
y_{n}+r_{n} & =\left(y_{n-1}+r_{n-1}\right)+a_{n-1} p^{n-1}+c_{n-1} p^{n-1} \\
& =\left(\sum_{k=0}^{n-2} \ell_{k}^{\prime} p^{k}+e_{n-1} p^{n-1}\right)+a_{n-1} p^{n-1}+c_{n-1} p^{n-1} \\
& =\left(\sum_{k=0}^{n-1} \ell_{k}^{\prime} p^{k}\right)+e_{n} p^{n},
\end{aligned}
$$

where the last equality holds by virtue of (4.2), using $c_{f}=c_{n-1}$ and $\ell_{1}=a_{n}$. This completes the induction step, proving (4.3).

Now the lifted path data yields the $p$-adic limit

$$
\lim _{n \rightarrow \infty} \sum_{k=0}^{n-1} \ell_{k}^{\prime} p^{k}=\lim _{n \rightarrow \infty}\left(y_{n}+r_{n}\right)-\lim _{n \rightarrow \infty} e_{n} p^{n}=y+r .
$$

We conclude that the lifted path of $\mathscr{G}^{\prime}$ corresponding to $y \in Y$ determines the point $y^{\prime} \stackrel{\text { def }}{=} y+r \in Y^{\prime}$. It follows that $Y^{\prime}=Y+r$, as asserted. This completes the argument in the case that $r$ has a purely periodic $p$-adic expansion.

It remains to treat the general case where $r$ has a preperiodic part, say length $Q_{0}$. We must extend the construction above and upper bound the number of states in the constructed presentation $(\mathcal{G}, \boldsymbol{w})$. The extension is routine: we add extra vertices $\boldsymbol{w} \stackrel{\text { def }}{=}\left(v, d_{j}, e, a\right)$ to $\mathscr{E}$, in which $v$ denotes a vertex of $\mathscr{E}, d_{j}$ marks the $j$-th preperiodic digit of $r, 1 \leq j \leq Q_{0}$. The exit edges of $\mathscr{G}^{\prime}$ map a vertex to $\boldsymbol{w}^{\prime}=\left(v^{\prime}, d_{j+1}, e^{\prime}, a^{\prime}\right)$ in which there is a directed labeled edge $\left(v, v^{\prime}\right) \in \mathcal{E}$, with label $\ell_{1}$ satisfying $\ell_{1}=a^{\prime}$. The edge label $\ell^{\prime}$ assigned to this edge will be $0 \leq \ell \leq p-1$ with

$$
\ell^{\prime} \equiv e+\ell_{1}+d_{j}(\bmod p)
$$

and the value $e^{\prime}$ is required to satisfy

$$
e^{\prime}=\frac{1}{p}\left(e+\ell_{1}+d_{j}-\ell^{\prime}\right) .
$$

The final preperiodic digit exit edges go to vertices $\boldsymbol{w} \stackrel{\text { def }}{=}\left(v^{\prime}, f_{0}, e^{\prime}, a^{\prime}\right)$ in the earlier set.

It is straightforward to check that the underlying labeled graph has the rightresolving property. Next one must check that this extension preserves the lifting property of paths, we omit the details.

Finally we must upper bound the total number of vertices in the graph $(\mathcal{E}, \boldsymbol{w})$. One finds that the preperiodic part contributes at most $2 Q_{0} V p$ vertices, and the periodic part contributes at most $2 Q V p$ vertices. 
Remark 4.2. The key features in this proof are: (i) the $p$-adic carry digits propagate to higher powers of $p$ and do not disturb earlier $p$-adic digits; (ii) the size of the carry digits is bounded above. Property (i) fails in real number arithmetic, and there is no real number analogue of this result.

4.2. Minkowski sum of two $p$-adic path sets. We show that the Minkowski sum of two $p$-adic path set fractals is itself a path set, establishing Theorem 1.3. This proof is constructive, but it no longer produces a right-resolving presentation.

Proof of Theorem 1.3. We suppose that $Y_{1} \stackrel{\text { def }}{=}\left(\bar{l}_{p}, \boldsymbol{E}_{1}, v_{1}\right)$ and $Y_{2} \stackrel{\text { def }}{=}\left(\bar{l}_{p}, \boldsymbol{E}_{2}, v_{2}\right)$ come with standard presentations. We use these presentations to directly construct a presentation $Y^{\prime} \stackrel{\text { def }}{=}\left(\bar{l}_{p}, \mathcal{E}_{1,2}, w_{0}\right)$, which is not necessarily standard, and show that $Y^{\prime}=Y_{1}+Y_{2}$, the Minkowski sum, certifying membership in $\ell\left(\mathbb{Z}_{p}\right)$.

To begin the construction, $\mathscr{\ell}_{1,2}$ will have vertices labeled $\boldsymbol{w} \stackrel{\text { def }}{=}\left(v_{j, 1}, v_{k, 2}, e, a\right)$ where $v_{j, 1} \in V\left(\mathscr{G}_{1}\right), v_{k, 2} \in V\left(\mathscr{G}_{2}\right), e \geq 0$ is an integer encoding carry-digit information, and $0 \leq a \leq p-1$ specifies an allowed edge entry label in $\mathscr{\ell}_{1,2}^{+}$.

The exit edges from a given vertex $\boldsymbol{w}$ go to a new vertex $\boldsymbol{w}^{\prime}=\left(v_{j^{\prime}, 1}, v_{k^{\prime}, 2}, e^{\prime}, a^{\prime}\right)$ in which

(a) there is a directed edge of $\ell_{1}$ from $v_{j, 1}$ to $v_{j^{\prime}, 1}$ having label $\ell_{1}$ satisfying $\ell_{1}=a^{\prime}$;

(b) a directed edge of $\mathscr{E}_{2}$ from $v_{k, 2}$ to $v_{k^{\prime}, 2}$ with label $\ell_{2}$;

(c) the constructed edge is assigned the label $\ell, 0 \leq \ell \leq p-1$, determined by

$$
\ell \equiv e+\ell_{1}+\ell_{2}(\bmod p), \quad 0 \leq \ell \leq p-1
$$

(d) the new carry-digit is

$$
e^{\prime}=\frac{1}{p}\left(e+\ell_{1}+\ell_{2}-\ell\right) \geq 0 .
$$

The initial pointed vertex of the graph $\mathscr{E}_{1,2}$ is $\boldsymbol{w}_{0} \stackrel{\text { def }}{=}\left(v_{0,1}, v_{0,2}, 0,0\right)$. We now define $\boldsymbol{E}_{1,2}$ to consist of all vertices above reachable from $\boldsymbol{w}_{0}$ by some directed path. We show this is a finite graph by establishing that that the "carry-digit" in any reachable vertex satisfies $e \leq 2$. This follows by induction on the length of the path. The base case is $n=0$ where $e=0$. For the induction step, we upper bound the new value of $e$ via

$$
e^{\prime}=\frac{1}{p}\left(e+\ell_{1}+\ell_{2}-\ell\right) \leq \frac{1}{p}(2+(p-1)+(p-1)) \leq 2,
$$

completing the induction step. We then insert all edges between these vertices produced in the construction above.

To see that $Y_{1}^{\prime}=Y_{1}+Y_{2}$, we prove by induction on $n \geq 0$ that being at a vertex $\boldsymbol{w}$ at step $n$, having gotten a specified series of edge labels, following a given lifted path implies that 
(1) the steps and vertices of the lifted path have sufficient information to reconstruct two paths of input $y_{1}$ and $y_{2}$ producing that path;

(2) the first $n p$-adic digit symbols of $y_{1}+y_{2}$ have been correctly computed by symbols of the steps of the path so far, namely that if

$$
y_{i, n}=\sum_{k=0}^{n-1} a_{k, i} p^{k}, \quad i=1,2,
$$

then

$$
y_{1, n}+y_{2, n}=\sum_{k=0}^{n-1} b_{k} p^{k}+e p^{n},
$$

where $e=e_{n}$ is the current carry-digit, and the $b_{i}$ are the edge labels produced so far in the graph $\mathscr{E}_{1,2}^{+}$.

Suppose that the next directed edge moves to a vertex $\boldsymbol{w}^{\prime}=\boldsymbol{w}_{n+1}$, with data $\left(e^{\prime}, a^{\prime}\right)$. Then we have $a^{\prime}=\ell_{1}=a_{n, 1}$ and

$$
e^{\prime}=\frac{1}{p}\left(\ell_{1}+\ell_{2}-\ell+e\right)=\frac{1}{p}\left(a+\ell_{2}-\ell+e\right)
$$

Since $e, a, \ell$ are known, this equation uniquely determines the label $\ell_{2}=a_{n, 2}$. Since both $\mathscr{E}_{1}$ and $\mathscr{E}_{2}$ are right-resolving, the edges $\left(j, j^{\prime}\right)$ and $\left(k, k^{\prime}\right)$ with the labels $a_{n_{1}}, a_{n, 2}$ are legal steps which uniquely determine the edges updating $y_{1, n}, y_{2, n}$ to $y_{1, n+1}, y_{2, n+1}$. Now the definition of edge labels in $\mathcal{G}_{1,2}$ assigns the label $b_{n} \stackrel{\text { def }}{=} \ell$ to the edge of $\mathscr{G}_{1,2}$ and $e^{\prime}=e_{n+1}$ in

$$
y_{1, n+1}+y_{2, n+1}=\sum_{k=0}^{n} b_{k} p^{k}+e^{\prime} p^{n},
$$

completing the induction step.

Remark 4.3. The presentation $Y_{1}+Y_{2}=\left(\bar{l}_{p}, \boldsymbol{g}_{1,2}^{+}, \boldsymbol{w}_{0}\right)$ in this construction is generally far from right-resolving. This occurs because some values $y=y_{1}+y_{2} \in$ $Y_{1}+Y_{2}$ may have more than one representation $\left(y_{1}, y_{2}\right)$. This construction produces a separate path for each pair $\left(y_{1}, y_{2}\right)$, so more than one path can yield the same sequence of labels.

\section{5. $p$-adic multiplication and path set fractals}

5.1. Multiplication by $\boldsymbol{p}$-integral rational numbers. We give constructive proof for multiplication by rational numbers of specific types. 
Theorem 5.1. Let $Y$ belong to $\varrho\left(\mathbb{Z}_{p}\right)$ and suppose it has a standard presentation $\left(\bar{l}_{p}, \mathcal{E}, v_{0}\right)$ having $V$ vertices. Let $M \geq 2$ be a positive integer with $\operatorname{gcd}(p, M)=1$.

(1) For $r=M$ the multiplicatively shifted set $\left.Y^{\prime} \stackrel{\text { def }}{=} M Y \in \mathbb{Z}_{p}\right)$. It has a right-resolving presentation having at most $(M+1) V$ vertices.

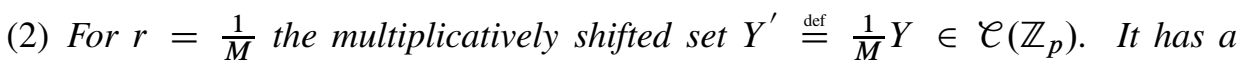
right-resolving presentation having at most $(M+1) V$ vertices.

(3) For $r=-1$ the multiplicatively shifted set $Y^{\prime} \stackrel{\text { def }}{=}-Y \in \ell\left(\mathbb{Z}_{p}\right)$. It has a rightresolving presentation having at most $2 \mathrm{~V}$ vertices.

(4) For $r=p^{k}, k \geq 0$, the multiplicatively shifted set $Y^{\prime} \stackrel{\text { def }}{=} p^{k} Y \in \bigodot\left(\mathbb{Z}_{p}\right)$. It has a right-resolving presentation having at most $k+V$ vertices.

Proof. We are given $Y \in \mathcal{C}\left(\mathbb{Z}_{p}\right)$, with a standard presentation $Y=\left(\bar{l}_{p}, \boldsymbol{E}, v_{0}\right)$, in which $\mathcal{E}$ has $V$ vertices. For each given $r$ we give an explicit construction of a standard presentation $Y^{\prime} \stackrel{\text { def }}{=}\left(\bar{l}_{p}, \boldsymbol{e}^{\prime}, \boldsymbol{w}_{0}\right)$ and establish that $Y^{\prime}=r Y$ in each case. The constructions in cases (1)-(3) are similar.

(1) Here $r=M$ with $p \nmid M$, and we construct a presentation $Y^{\prime}$ of a $p$-adic path set fractal and show $Y^{\prime}=M Y$. We start with an (infinite) graph $\mathcal{\ell}^{\prime \prime}$ whose vertices will be pairs $\boldsymbol{w}=(v, e)$, in which $v$ is a vertex of $\mathcal{G}$, and $e \geq 0$ is a carry-digit. The initial vertex is $\boldsymbol{w}_{0} \stackrel{\text { def }}{=}\left(v_{0}, 0\right)$. The exit edges from a vertex $\boldsymbol{w}$ to a vertex $\boldsymbol{w}^{\prime}=\left(v^{\prime}, e^{\prime}\right)$ will occur only if there is at least one edge from $v$ to $v^{\prime}$. Given such an edge of $\mathcal{E}$ with label $\ell$, we assign a corresponding edge of $\ell^{\prime}$ with label $\ell^{\prime}$ given by

$$
\ell^{\prime}=M \ell+e(\bmod p), \quad 0 \leq \ell^{\prime} \leq p-1,
$$

which is well-defined since $(p, M)=1$. We require that the new carry digit be

$$
e^{\prime} \stackrel{\text { def }}{=} \frac{1}{p}\left(e+M \ell-\ell^{\prime}\right) .
$$

We define $\left(\boldsymbol{E}^{\prime}, \boldsymbol{w}_{0}\right)$ to be the graph obtained by taking all vertices reachable from $\boldsymbol{w}_{0}$ in the above construction. We prove that all reachable vertices have carry-digit $0 \leq e \leq M$ by induction on the number of steps $n$ on a minimal path to such a vertex. The base case $n=0$ is true, since $e=0$, and the induction step follows by observing from (5.2) that

$$
e^{\prime} \leq \frac{1}{p}(M+M(p-1)) \leq M
$$

We conclude that the graph $\mathscr{\ell}^{\prime}$ has at most $(M+1) V$ vertices.

We now set $Y^{\prime} \stackrel{\text { def }}{=}\left(\bar{l}_{p}, \mathcal{E}^{\prime}, \boldsymbol{w}_{0}\right)$, and first show this presentation is standard. We first claim that $\mathcal{E}^{\prime}$ is right-resolving. We argue by contradiction. If not, there would be two exit edges of some vertex $\boldsymbol{w}=(v, e)$ of $\mathcal{E}$ having the same value of $\ell^{\prime}$. But then by $(5.1)$ the underlying edges of $\left(\mathcal{S}, v_{0}\right)$ would have the same value of $\ell$, contradicting 
the right-resolving property of $\left(\mathscr{G}, v_{0}\right)$. By construction $\left(\mathscr{G}^{\prime}, \boldsymbol{w}_{0}\right)$ is reachable, hence this presentation of $Y^{\prime}$ is standard.

It remains to show that $Y^{\prime}=M Y$. Consider an infinite path in $\mathcal{E}$, with image

$$
y_{\infty}=\sum_{j=0}^{\infty} \ell_{j} p^{j} \in Y .
$$

We assert that the corresponding output path

$$
y_{\infty}^{\prime}=\sum_{j=1}^{\infty} \ell_{j}^{\prime} p^{j} \in Y^{\prime}
$$

has

$$
y_{\infty}^{\prime}=\frac{1}{M} y_{\infty} .
$$

We verify this by induction on the length of a finite path approximating $y_{\infty}$. Let $v_{0}, v_{1}, \ldots, v_{n}$ be states on a path in $s G$ with edge labels $\ell_{0}, \ell_{1}, \ldots, \ell_{n-1}$. Associated to this path is

$$
y_{n}=\ell_{0}+\ell_{1} p+\cdots+\ell_{n-1} p^{n-1} .
$$

By construction we obtain

$$
y_{n}^{\prime}=\sum_{j=0}^{n-1} \ell_{j}^{\prime} p^{j} .
$$

Now we prove by induction on $n \geq 1$ that

$$
y_{n}^{\prime}=M y_{n}+e_{n} p^{n},
$$

where $e_{n}=e^{\prime}$, where $e^{\prime}$ is the carry value at the final vertex $v_{n}$. The base case $n=1$ is clear, and for the induction step $\left(e_{n}=e, e_{n+1}=e^{\prime}\right)$ we get, using (5.2),

$$
M y_{n+1}=M\left(y_{n}+\ell_{n} p^{n}\right)=y_{n}^{\prime}+\left(e_{n}+M \ell_{n}\right) p^{n}=y_{n}^{\prime}+e_{n+1} p^{n+1} .
$$

Now we use $\left|e_{n} p^{n}\right|_{p} \rightarrow 0$ as $n \rightarrow \infty$, whence $M y_{\infty}^{\prime}=y_{\infty}$, establishing the result.

(2) Here $r=\frac{1}{M}$ with $p \nmid M$, and we construct a presentation $Y=\left(\bar{l}_{p}, \boldsymbol{E}^{\prime}, \boldsymbol{w}_{0}\right)$ of $\frac{1}{M} Y$. We start with an infinite graph $\mathscr{E}_{M}$, whose vertices will be pairs $\boldsymbol{w}=(v, e)$, in which $v$ is a vertex of $\mathscr{E}$, and $e \geq 0$ is a carry digit, initially unbounded. The initial vertex is $\boldsymbol{w}_{0} \stackrel{\text { def }}{=}\left(v_{0}, 0\right)$. The exit edges from a vertex $\boldsymbol{w}=(v, e)$ to a vertex $\boldsymbol{w}=\left(v^{\prime}, e^{\prime}\right)$ will occur only if there is at least one edge in $\mathscr{E}$ from $v$ to $v^{\prime}$. Given such an edge of $\mathcal{E}$ with label $\ell$, we assign a corresponding edge of $\mathcal{E}^{\prime}$ with label $\ell^{\prime}$ given by

$$
M \ell^{\prime}=\ell-e(\bmod p), \quad 0 \leq \ell^{\prime} \leq p-1,
$$


which is well-defined since $(p, M)=1$. We require that the new carry digit be

$$
e^{\prime} \stackrel{\text { def }}{=} \frac{1}{p}\left(e+M \ell^{\prime}-\ell\right)
$$

We now define $\left(\mathscr{G}^{\prime}, \boldsymbol{w}_{0}\right)$ to be the graph obtained by including only the vertices reachable from $\boldsymbol{w}_{0}$ in the above construction. We now show all reachable vertices have carry-digit $0 \leq e \leq M$, by induction on the number of steps $n$ on a minimal path to such a vertex. The base case $n=0$ holds since $e=0$, and the induction step follows by observing from (5.4) that

$$
e^{\prime} \leq \frac{1}{p}(M+M(p-1)) \leq M .
$$

We conclude that the graph $\mathcal{E}^{\prime}$ has at most $(M+1) V$ vertices.

We now define the $p$-adic path set fractal $Y^{\prime} \stackrel{\text { def }}{=}\left(\bar{l}_{p}, \mathscr{E}^{\prime}, \boldsymbol{w}_{0}\right)$, and first show this presentation is standard. To show $\mathscr{E}^{\prime}$ is right-resolving, we argue by contradiction. If not, there would be two exit edges of some vertex $\boldsymbol{w}=(v, e)$ of $\mathcal{E}$ having the same value of $\ell^{\prime}$. But then by (5.3) the underlying edges of $\left(\mathscr{\xi}, v_{0}\right)$ would have the same value of $\ell$, contradicting the right-resolving property of $\left(\mathscr{E}, v_{0}\right)$. It is reachable by construction, so it is a standard presentation.

It remains to establish that $Y^{\prime}=\frac{1}{M} Y$, still supposing $(p, M)=1$. Consider an infinite path in $\mathcal{E}$, with image

$$
y_{\infty}=\sum_{j=0}^{\infty} \ell_{j} p^{j} \in Y .
$$

We assert that the corresponding output path

$$
y_{\infty}^{\prime}=\sum_{j=1}^{\infty} \ell_{j}^{\prime} p^{j} \in Y^{\prime}
$$

has

$$
y_{\infty}^{\prime}=\frac{1}{M} y_{\infty}
$$

We verify this by induction on the length of a path starting from the initial vertex. Let $v_{0}, v_{1}, \ldots, v_{n}$ be vertices on a path in $\mathcal{E}$ with edge labels $\ell_{0}, \ell_{1}, \ldots, \ell_{n-1}$. Associated to this path is

$$
y_{n}=\ell_{0}+\ell_{1} p+\cdots+\ell_{n-1} p^{n-1} .
$$

By construction we obtain

$$
y_{n}^{\prime}=\sum_{j=0}^{n-1} \ell_{j}^{\prime} p^{j} .
$$


Now we prove by induction that

$$
M y_{n}^{\prime}=y_{n}+e_{n} p^{n}
$$

where $e_{n}=e^{\prime}$ is the carry value at the final vertex $v_{n}$. For the induction step $\left(e_{n}=e, e_{n+1}=e^{\prime}\right)$ we get, using (5.4)

$$
M y_{n+1}^{\prime}=M\left(y_{n}^{\prime}+\ell_{n}^{\prime} p^{n}\right)=y_{n}+\left(e_{n}+M \ell_{n}^{\prime}\right) p^{n}=y_{n+1}+e_{n+1} p^{n+1} .
$$

Now we use $\left|e_{n} p^{n}\right|_{p} \rightarrow 0$ as $n \rightarrow \infty$, whence $M y_{\infty}^{\prime}=y_{\infty}$ establishing the result.

(3) Given a standard presentation of $Y \stackrel{\text { def }}{=}\left(\bar{l}_{p}, \mathcal{E}, v_{0}\right)$, we construct a standard presentation $Y^{\prime} \stackrel{\text { def }}{=}\left(\bar{l}_{p}, \mathcal{E}^{\prime \prime}, \boldsymbol{w}_{0}\right)$ which has $Y^{\prime} \stackrel{\text { def }}{=}-Y$, as follows.

The vertices of $\mathcal{E}^{\prime}$ will be pairs $\boldsymbol{w}=(v, e)$, in which $v$ is a vertex of $\mathcal{G}$, and $e$ is a carry digit, which may take values 0 or -1 . The initial vertex will be $\boldsymbol{w}_{0} \stackrel{\text { def }}{=}\left(v_{0}, 0\right)$. The exit edges from a vertex $\boldsymbol{w}$ to a vertex $\boldsymbol{w}^{\prime}=\left(v^{\prime}, e^{\prime}\right)$ will occur only if there is at least one edge in $\mathcal{G}$ from $v$ to $v^{\prime}$. Given such an edge of $\mathcal{G}$ with label $\ell$, we assign to it a corresponding edge of $\mathscr{\ell}^{\prime}$ from $\boldsymbol{w}$ to $\boldsymbol{w}^{\prime}$ with label $\ell^{\prime}$ given by

$$
\ell^{\prime}=-\ell+e(\bmod p), \quad 0 \leq \ell^{\prime} \leq p-1,
$$

If the current vertex has $e=0$ and $\ell=0$, then the new vertex has $\ell^{\prime}=0$ and is assigned carry digit $e^{\prime}=0$. If either $e=-1$ or $e=0$ and $\ell>0$, then the new carry digit $e^{\prime}=-1$. Once a path in $\ell^{\prime}$ reaches a vertex with carry digit $e^{\prime}=-1$, all subsequent vertices reached have carry digit -1 . Note that when $e=-1$ we have $-p \leq-\ell-1 \leq-1$ and $\ell^{\prime}=p-\ell-1$.

We now let $\mathscr{G}^{\prime}$ denote the part of the graph above reachable from the initial vertex $\boldsymbol{w}_{0}$. This graph has at most $2 V$ vertices. We then insert all edges between reachable vertices produced in the construction above.

We now set $Y^{\prime} \stackrel{\text { def }}{=}\left(\bar{l}_{p}, \boldsymbol{E}^{\prime}, \boldsymbol{w}_{0}\right)$, and as before check that this is a standard presentation. We claim that $\mathscr{\ell}^{\prime}$ is right-resolving. This is clear since the label $\ell^{\prime}$ on exit edges from a vertex $\boldsymbol{w}$ are in one-one correspondence with labels on exit edges in $\mathcal{E}$ from the associated vertex $v$, via (5.5). The graph $\mathcal{G}^{\prime}$ is reachable by construction.

It remains to show that $Y^{\prime}=-Y$. We let $y_{n}=\sum_{j=0}^{n-1} \ell_{j} p^{j}$ and

$$
y_{n}^{\prime}=\sum_{j=0}^{n-1} \ell_{j} p^{j}
$$

We have $y_{n}^{\prime}=y_{n}=0$ as long as the carry digit $e=0$. Let $\ell_{r}-1$ be the first nonzero digit on the path, where the carry digit switches to -1 . From then on switches to $e=-1$, we have 


$$
y_{n}^{\prime}=\left(p-\ell_{r-1}\right) p^{r-1}+\sum_{j=r}^{n-1}\left(p-\ell_{j}-1\right) p^{j}=-y_{n}+p^{n} .
$$

Letting $n \rightarrow \infty$ we obtain $y_{\infty}^{\prime}=-y_{\infty}$, establishing the result.

(4) Let $r=p^{k}$ with $n \geq 1$. For $k \geq 0$ the set $p^{k} Y$ consists of modifying all symbol sequences in $Y$ by adding $k$ initial zeros. A standard presentation $Y^{\prime}=$ $\left(\bar{l}_{p}, \mathscr{E}^{\prime}, \boldsymbol{w}\right)$ for this set is easily obtained. Let $\mathscr{G}^{\prime}$ consist of $\mathcal{G}$ with the addition of $k$ new vertices $\boldsymbol{w}_{j}(0 \leq j \leq k-1)$. Each of the new vertices has a single exit edge from $\boldsymbol{w}_{j}$ to $\boldsymbol{w}_{j+1}$ assigned label 0 , for $0 \leq j \leq k-2$, and a similar exit edge labeled 0 from $\boldsymbol{w}_{k-1}$ to $v_{0}$. The start vertex of $\mathscr{\ell}^{\prime}$ is $\boldsymbol{w}_{0}$, and $\mathscr{\ell}^{\prime}$ has $k+V$ vertices.

Remark 5.2. Theorem 5.1 excluded the case "multiplication by $p^{k}$ with $k<0$," since these maps do not have range in $\mathbb{Z}_{p}$.

5.2. Proof of Theorem 1.4. Theorem 1.4 follows immediately from Theorem 5.1.

Proof of Theorem 1.4. Let $r$ be a $p$-integral rational number, i.e. $\operatorname{ord}_{p}(r) \geq 0$. We may factor $r=(-1)^{a} p^{k} \frac{M_{1}}{M_{2}}$, in which $a \in\{0,1\}, k \geq 0$ and $\operatorname{gcd}\left(p, M_{1} M_{2}\right)=1$. Now we successively apply the constructions in Theorem 5.1 to multiply $Y$ by $\frac{1}{M_{1}}$, next multiply the resulting set by $M_{2}$, next multiply the resulting set by $(-1)^{a}$, and finally multiply the resulting set by $p^{k}$.

\section{Examples}

In the following examples, we let $\Sigma_{p}(D)$ denote the $p$-adic integer Cantor set consisting of all $p$-adic integers whose digits are drawn from a given set $D \subseteq$ $\{0,1, \ldots, p-1\}$. All $\Sigma_{p}(D) \in \mathcal{C}\left(\mathbb{Z}_{p}\right)$, and have Hausdorff dimensions

$$
d_{H}\left(\Sigma_{p}(D)\right)=\log _{p}|D|=\frac{\log |D|}{\log p} .
$$

Example 6.1. This example concerns adding a $p$-integral rational number $r$ to the 3 -adic Cantor set $Y_{01} \stackrel{\text { def }}{=} \Sigma_{3}(\{0,1\})$, whose 3 -adic expansions omit the digit 2 . It has a right-resolving presentation as a 3 -adic path set by the pointed labeled graph $(\mathscr{G}, 0)$ pictured in Figure 1.

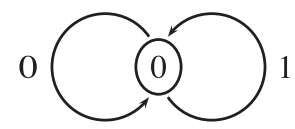

Figure 1. Presentation $(\mathscr{E}, 0)$ of $\Sigma_{3}(\{0,1\})$. 
This graph has adjacency matrix

$$
\boldsymbol{A}=\text { (2), }
$$

whose Perron-Frobenius eigenvalue is 2 , hence the Cantor set $\Sigma_{3}(\{0,1\})$ has Hausdorff dimension $d_{H}\left(\Sigma_{3}(\{0,1\})\right)=\log _{3} 2$, as stated above.

Now we consider the effect of additively shifting by $r=2$. The construction of Section 4.1 applied to the presentation above yields the $p$-adic path set fractal presentation of $Y_{01}+2=\Sigma_{3}(\{0,1\})+2$, given in Figure 2, denoted by

$$
Y_{01}+2=i_{3}\left(X \mathscr{E}^{\prime}(0200)\right) \text {. }
$$

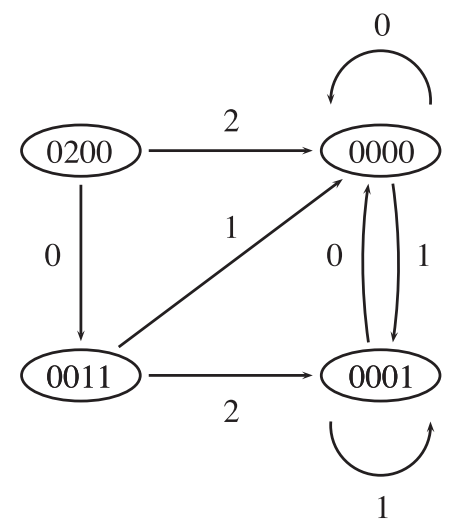

Figure 2. Presentation $\left(\mathscr{G}^{\prime}, 0200\right)$ of $Y_{01}+2$.

Under one ordering of the vertices of $\mathscr{E}^{\prime}$, the adjacency matrix of the underlying (undirected) graph of $\mathscr{G}^{\prime}$ is

$$
\boldsymbol{A}^{\prime}=\left(\begin{array}{llll}
0 & 1 & 1 & 0 \\
0 & 0 & 1 & 1 \\
0 & 0 & 1 & 1 \\
0 & 0 & 1 & 1
\end{array}\right)
$$

The eigenvalues of $\boldsymbol{A}^{\prime}$ are 2 and 0 (multiplicity 3 ), so we see the Perron eigenvalue is 2 . Thus the Hausdorff dimension is

$$
d_{H}\left(Y_{01}+2\right)=\frac{\log 3}{\log 2}=d_{H}\left(Y_{01}\right)
$$

Here $Y_{01}+\alpha$ for any $\alpha \in \mathbb{Z}_{3}$ must have the same Hausdorff dimension, because they are bi-Lipschitz equivalent, hence their adjacency matrices must have the same Perron eigenvalue. Note that only a countable set of values of $\alpha$ can give $Y_{01}+\alpha \in \mathscr{C}\left(\mathbb{Z}_{3}\right)$, since $\mathcal{C}\left(\mathbb{Z}_{3}\right)$ is a countable set. 
Example 6.2. We consider the effect of set addition on 5 -adic Cantor sets $\Sigma_{5}(D)$ for certain subsets of digits $D$. For all sets of two digits, we have $d_{H}\left(\Sigma_{5}(\{a, b\})=\right.$ $\log _{5} 2$. Set

$$
Y_{i, j} \stackrel{\text { def }}{=} \Sigma_{5}(\{0, i\})+\Sigma_{5}(\{0, j\}), \quad 1 \leq i, j \leq 4 .
$$

One can show that

$$
\log _{5} 3 \leq d_{H}\left(X_{i, j}\right) \leq \log _{5} 4 .
$$

We find by inspection that the sums of certain Cantor sets are themselves Cantor sets:

$$
Y_{1,1} \stackrel{\text { def }}{=} \Sigma_{5}(\{0,1\})+\Sigma_{5}(\{0,1\})=\Sigma_{5}(\{0,1,2\})
$$

and

$$
Y_{1,2} \stackrel{\text { def }}{=} \Sigma_{5}(\{0,1\})+\Sigma_{5}(\{0,2\})=\Sigma_{5}(\{0,1,2,3\}) .
$$

These examples have Hausdorff dimensions $d_{H}\left(Y_{1,1}\right)=\log _{5} 3$ and $d_{H}\left(Y_{1,2}\right)=$ $\log _{5} 4$, respectively, and they show that the bounds in (6.1) are sharp. Much more interesting are the sets $Y_{2,3}$ and $Y_{1,4}$, which are not Cantor sets; here the $p$-adic carry operations occurring during addition in the set sum destroy the Cantor set property. To compute their Hausdorff dimension, we first find $p$-adic path set presentations for them by the construction of Theorem 1.3. These presentations are not right-resolving, but we then apply the subset construction method in [2], Section 2, to obtain a rightresolving presentation. We omit the details, noting only that for $Y_{1,4}$ we find the resulting graph has five vertices and adjacency matrix

$$
A_{14}=\left(\begin{array}{ccccc}
1 & 1 & 1 & 0 & 0 \\
1 & 1 & 1 & 0 & 0 \\
1 & 1 & 0 & 1 & 1 \\
1 & 1 & 0 & 1 & 1 \\
1 & 1 & 0 & 1 & 1
\end{array}\right)
$$

Its Perron eigenvalue is $2+\sqrt{2}$. Computing its Hausdorff dimension by the formula of Theorem 3.1, we obtain

$$
d_{H}\left(Y_{1,4}\right)=\log _{5}(2+\sqrt{2}) \approx \log _{5}(3.41412) .
$$

A similar construction for $Y_{2,3}$ leads to

$$
d_{H}\left(Y_{2,3}\right)=\log _{5}(2+\sqrt{3}) \approx \log _{5}(3.73205) .
$$

Example 6.3. We consider the effect on the 3 -adic Cantor set $Y_{01} \stackrel{\text { def }}{=} \Sigma_{3}(\{0,1\})$ of a multiplicative translation by $r=\frac{1}{4}$. The set $\frac{1}{4} Y_{01}$ has a presentation $(\mathscr{H}, 00)$ obtained from that of $\Sigma_{3}(\{0,1\})$ given by $(\mathscr{G}, 0)$, using the construction given in Section 5.1. This presentation is shown in Figure 3. 


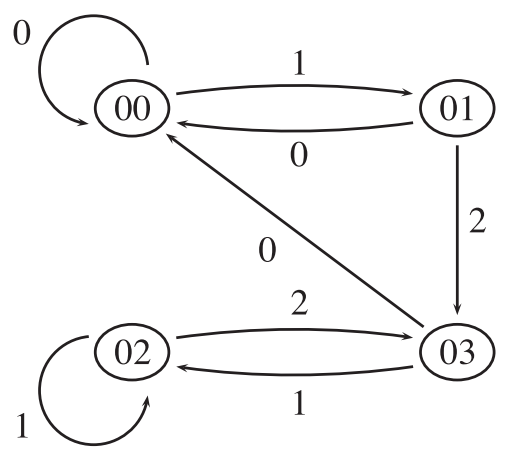

Figure 3. Presentation $(\mathscr{H}, 00)$ of $\frac{1}{4} \Sigma_{3}(\{0,1\})$.

The adjacency matrix $\boldsymbol{B}$ of the underlying graph of $\mathscr{H}$ is

$$
\boldsymbol{B}=\left(\begin{array}{llll}
1 & 1 & 0 & 0 \\
1 & 0 & 0 & 1 \\
0 & 0 & 1 & 1 \\
1 & 0 & 1 & 0
\end{array}\right)
$$

This matrix has Perron eigenvalue 2, and it has three other smaller nonzero eigenvalues, one real and two conjugate complex. Using the formula in Theorem 3.1(3) we obtain $d_{H}\left(\frac{1}{4} Y_{01}\right)=\log _{3} 2$.

Example 6.4. In this example we consider the effect of intersecting multiplicatively translated Cantor sets taken from Example 6.3. Let

$$
Y \stackrel{\text { def }}{=} \frac{1}{4} Y_{01} \cap Y_{01}=\Sigma_{3}(\{0,1\}) \cap \frac{1}{4} \Sigma_{3}(\{0,1\}) .
$$

We obtain by the method of [2], Section 4, applied to the presentations above the presentation of $\left(\mathscr{H}^{\prime}, 000\right)$ shown in Figure 4 , where $\mathscr{H}^{\prime}$ is the label product $\mathscr{H}^{\prime}=$ $\mathcal{H} \star \mathcal{H}$, as defined in [2], Section 4 .

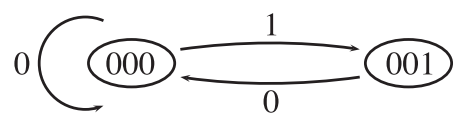

Figure 4. Presentation $\left(\mathscr{H}^{\prime}, 000\right)$ of $\Sigma_{3}(\{0,1\}) \cap \frac{1}{4} \Sigma_{3}(\{0,1\})$.

The adjacency matrix of the underlying graph of $\mathscr{H}^{\prime}$ is

$$
\boldsymbol{B}^{\prime}=\left(\begin{array}{ll}
1 & 1 \\
1 & 0
\end{array}\right),
$$


whose Perron eigenvalue is $\frac{1+\sqrt{5}}{2}$. We conclude that

$$
d_{H}(Y)=d_{H}\left(\Sigma_{3}(\{0,1\}) \cap \frac{1}{4} \Sigma_{3}(\{0,1\})\right)=\log _{3}\left(\frac{1+\sqrt{5}}{2}\right) .
$$

Remark 6.5. In [3] we will study intersections of multiplicative translates of 3-adic Cantor sets in much more detail.

\section{Concluding remarks}

The constructions of this paper may prove interesting from the viewpoint of nonnegative integer matrices and their eigenvalues. By Theorem 1.1 the Hausdorff dimension is given by the base $p$ logarithm of the spectral radius of the underlying adjacency matrix of a standard path set presentation graph, which is a nonnegative integer matrix. As noted in Section 1.2, for nonzero $r \in \mathbb{Q} \cap \mathbb{Z}_{p}$ the maps $X \mapsto X+r$ and $X \mapsto r X$ preserve Hausdorff dimension. On the level of path set presentations these constructions therefore produce infinitely many different integer matrices, of varying dimensions, all having the same spectral radius, plus various eigenvalues of smaller modulus whose cardinality and size will change under these operations. The allowed dimension of these matrices as the parameter $r$ varies will be unbounded.

The spectral radius of a nonnegative matrix is always attained by a nonnegative real eigenvalue, according to the Perron-Frobenius theory. In the special case of nonnegative integer matrices $\boldsymbol{A}$ this maximal eigenvalue is a real algebraic integer $\beta$, and if $\boldsymbol{A}$ is not nilpotent, then $\beta \geq 1$. It is termed the Perron eigenvalue in Lind and Marcus [17], Definition 4.4.2. This eigenvalue is necessarily a weak Perron number, which is defined to be any positive $n$-th root of some Perron number ([16]) for some $n \geq 1$; a Perron number is any real algebraic integer $\beta \geq 1$ which is strictly larger in absolute value than all of its conjugates. Lind [15], Theorem 1, showed that the Perron eigenvalue of any aperiodic nonnegative integer matrix is a Perron number, and that conversely every Perron number occurs as the Perron eigenvalue of some aperiodic nonnegative integer matrix. More generally the Perron eigenvalue of any non-nilpotent nonnegative integer matrix is a weak Perron number, and conversely every weak Perron number occurs as the Perron eigenvalue of at least one such matrix. Perron numbers appear as the topological entropies of Axiom A diffeomorphisms via a result of Bowen [4]; see [15], p. 288.

The constructions in this paper could be of interest in investigating and producing examples of graphs with a fixed Perron eigenvalue, particularly in case where this eigenvalue is very close to 1 . In order to produce nonnegative matrices that have a given Perron number $\beta$ as spectral radius, it is sometimes necessary to take a nonnegative matrix of dimension strictly larger than the degree of the minimal polynomial of $\theta$, see an example given in Lind [14] and [15], Section 3. In such cases the characteristic polynomial of this matrix must contain extraneous eigenvalues. 
The constructions of this paper offer a method to generate interesting examples of this kind. Such graph constructions might also conceivably be useful in investigating conjectures on the smallest Perron number of each degree, a topic studied in Boyd [5] and $\mathrm{Wu}$ [24]. A more speculative direction would be to investigate the structure of such graphs in connection with Lehmer's conjecture on the Mahler measure of irreducible polynomials.

\section{References}

[1] A. G. Abercrombie, The Hausdorff dimension of some exceptional sets of $p$-adic integer matrices. J. Number Theory 53 (1995), 311-341. MR 1348767 Zbl 0837.11042

[2] W. Abram and J. C. Lagarias, Path sets in one-sided symbolic dynamics. To appear in Adv. in Appl. Math. Preprint 2012. arXiv:1207.5004

[3] W. Abram and J. C. Lagarias, Intersections of multiplicative translates of 3-adic Cantor sets. To appear in J. Fractal Geom. Preprint 2013. arXiv:1308.3133

[4] R. Bowen, Topological entropy and Axiom A. In Global Analysis (Proc. Sympos. Pure Math., Vol. XIV, Berkeley, Calif., 1968). American Mathematical Society, Providence, RI, 1970, 23-41. MR 262459 Zbl 0207.54402

[5] D. W. Boyd, The maximal modulus of an algebraic integer. Math. Comp. 45 (1985), 243-249 and S17-S20. MR 0790657 Zbl 0575.12002 Zbl 0575.12003 (supplements)

[6] M. Das and S.-M. Ngai, Graph-directed iterated function systems with overlaps. Indiana Univ. Math. J. 53 (2004), 109-134. MR 2048186 Zbl 1065.28003

[7] G. Edgar, Measure, topology and fractal geometry. Second edition. Undergraduate Texts in Mathematics. Springer, New York, 2008. MR 2356043 Zbl 1152.28008

[8] G. E. Edgar and J. Golds, A fractal dimension estimate for a graph-directed iterated function system of non-similarities. Indiana Univ. Math. J. 48 (1999), 429-447. MR 1722803 Zbl 0944.28008

[9] P. Erdős, Some unconventional problems in number theory. Math. Mag. 52 (1979), 67-70. MR 0527408 Zbl 0407.10001

[10] K. J. Falconer, Fractal geometry. Mathematical foundations and applications. Second edition. John Wiley \& Sons, Hoboken, NJ, 2003. MR 2118797 Zbl 1060.28005

[11] H. Furstenberg, Disjointness in ergodic theory, minimal sets, and a problem in Diophantine approximation. Math. Systems Theory 1 (1967), 1-49. MR 0213508 Zbl 0146.28502

[12] J. E. Hutchinson, Fractals and self similarity. Indiana Univ. Math. Journal 30 (1981), 713-747. MR 0625600 Zbl 0598.28011

[13] J. C. Lagarias, Ternary expansions of powers of 2. J. London Math. Soc. (2) 79 (2009), 562-588. MR 2506687 Zbl 1193.11008

[14] D. Lind, Entropies and factorizations of topological Markov shifts. Bull. Amer. Math. Soc. (N.S.) 9 (1983), 219-222. MR $0707961 \mathrm{Zbl} 0524.58034$

[15] D. Lind, The entropies of topological Markov shifts and a related class of algebraic integers. Ergodic Theory Dynam. Systems 4 (1984), 283-300. MR 766106 Zbl 0546.58035 Zbl 0534.58030 
[16] D. Lind, Entropies of automorphisms of a topological Markov shift. Proc. Amer. Math. Soc. 99 (1987), 589-595. MR 0875406 Zbl 0619.54030

[17] D. Lind and B. Marcus, An introduction to symbolic dynamics and coding. Cambridge University Press, Cambridge, 1995. MR 1369092 Zbl 1106.37301

[18] K. Mahler, Lectures on diophantine approximations I. g-adic numbers and Roth's theorem. Prepared from the notes by R. P. Bambah of the lectures given at the University of Notre Dame in the Fall of 1957. University of Notre Dame Press, Notre Dame, IN, 1961. MR 0142509 Zbl 0158.29903

[19] R. D. Mauldin and S. C. Williams, On the Hausdorff dimension of some graphs. Trans. Amer. Math. Soc. 298 (1986), 793-803. MR 0860394 Zbl 0603.28003

[20] R .D. Mauldin and S. C. Williams, Hausdorff dimension in graph directed constructions. Trans. Amer. Math. Soc. 309 (1988), 811-829. MR 0961615 Zbl 0706.28007

[21] S.-M. Ngai, F. Wang, and X. Dong, Graph-directed iterated function systems satisfying the generalized finite type condition. Nonlinearity 23 (2010), 2333-2350. MR 2672647 Zbl 1196.28009

[22] S.-M. Ngai and Y. Wang, Hausdorff dimension of self-similar sets with overlaps. J. London Math. Soc. 63 (2001), 655-672. MR 1825981 Zbl 1013.28008

[23] C. A. Rogers, Hausdorff measures. Reprint of the 1970 original. With a foreword by K. J. Falconer. Cambridge Mathematical Library. Cambridge University Press, Cambridge, 1998. MR 1692618 Zbl 0915.28002

[24] Q. Wu, The smallest Perron numbers. Math. Comp. 79 (2010), 2387-2394. MR 2684371 Zbl 1219.11158

Received November 10, 2013

William C. Abram, Department of Mathematics, Hillsdale College, Hillsdale, MI 49242, U.S.A.

E-mail: wabram@hilldale.edu

Jeffrey C. Lagarias, Department of Mathematics, University of Michigan, East Hall, Room 3086, 530 Church Street, Ann Arbor MI 48109-1043, U.S.A.

E-mail: lagarias@umich.edu 